\title{
Combined MR Data Acquisition of Multicontrast Images Using Variable Acquisition Parameters and $K$-Space Data Sharing
}

\author{
Ralf Mekle, Andrew F. Laine, and Ed X. Wu*
}

\begin{abstract}
A new technique to reduce clinical magnetic resonance imaging (MRI) scan time by varying acquisition parameters and sharing $k$-space data between images, is proposed. To improve data utilization, acquisition of multiple images of different contrast is combined into a single scan, with variable acquisition parameters including repetition time (TR), echo time (TE), and echo train length (ETL). This approach is thus referred to as a "combo acquisition." As a proof of concept, simulations of MRI experiments using spin echo (SE) and fast SE (FSE) sequences were performed based on Bloch equations. Predicted scan time reductions of $25 \%-50 \%$ were achieved for 2-contrast and 3-contrast combo acquisitions. Artifacts caused by nonuniform $k$-space data weighting were suppressed through semi-empirical optimization of parameter variation schemes and the phase encoding order. Optimization was assessed by minimizing three quantitative criteria: energy of the "residue point spread function (PSF)," energy of "residue profiles" across sharp tissue boundaries, and energy of "residue images." In addition, results were further evaluated by quantitatively analyzing the preservation of contrast, the PSF, and the signal-to-noise ratio. Finally, conspicuity of lesions was investigated for combo acquisitions in comparison with standard scans. Implications and challenges for the practical use of combo acquisitions are discussed.
\end{abstract}

Index Terms-Combined/combo acquisition, $k$-space data sharing, MRI, scan time reduction, variable acquisition parameter.

\section{INTRODUCTION}

$\mathbf{S}$ CAN TIME reduction in magnetic resonance imaging (MRI) remains an important issue, especially when considering acquisition of diagnostic images in a clinical setting. Shortenings of acquisition times yield reduction of costs and increased patient throughput and comfort. Improvements in scanner hardware over the last twenty years have aided in reducing scan time by allowing the development of fast acquisition schemes, such as echo planar imaging (EPI) [1], fast spin echo (FSE [2] or RARE [3]), and fast gradient echo

Manuscript received October 17, 2002; revised January 14, 2003. The work of E. X. Wu was supported in part by the National Institutes of Health (NIH) under Career Award CA85594. The Associate Editor responsible for coordinating the review of this paper and recommending its publication was M. W. Vannier. Asterisk indicates corresponding author.

R. Mekle is with the Department of Biomedical Engineering, Columbia University, New York, NY 10027 USA (e-mail: rm197@ columbia.edu).

A. F. Laine is with the Department of Biomedical Engineering and with the Department of Radiology, Columbia University, New York, NY 10027 USA.

*E. X. Wu is with the Department of Radiology, Columbia University, 710 W. 168th Street - Unit 63, New York, NY 10032 USA (e-mail: xew1@columbia.edu).

Digital Object Identifier 10.1109/TMI.2003.815054 sequences [4]. Most of these methods attempt to increase the amount of data acquired within one sequence or repetition time (TR) cycle, i.e., a larger portion of $k$-space is sampled before the next sequence cycle.

Other approaches to scan time reduction have included partial Fourier imaging [5], generalized series reconstruction [6], keyhole imaging [7], [8], reduced field-of-view (FOV) acquisition [9], adaptive dynamic MRI [10], unaliasing by Fourier-encoding the overlaps using the temporal dimension (UNFOLD) [11], and reception of MR signals with multiple coils (SMASH, SENSE) [12], [13]. Most of these methods aim at reducing the amount of $k$-space information that needs to be acquired. In addition, fast scans can also be designed by traversing $k$-space along different trajectories instead of a Cartesian grid [14], [15], such as spiral [16], radial [17], and "rosette-like" trajectories [18]. Furthermore, non-Fourier encoding techniques [19] offer another approach for increasing MR imaging efficiency. Finally, variable acquisition parameters have been employed in conventional MRI sequences to reduce scan time. In an early work [20], TR and TE were varied for the acquisition of high spatial frequency phase encoding (PE) views to achieve scan time reduction for obtaining a $T_{2}$-weighted image. Additional studies utilizing variable acquisition parameters did not necessarily focus on scan time reduction. For example, variable TE has been previously used to shorten the echo time for in vivo MR microscopy [21] and to reduce fat signal in three-dimensional time-of-flight MR angiography (MRA) [22]. However, the loss of small objects in variable TE imaging and its implications for FSE, RARE, and EPI were discussed in [23]. Similarly, the TR was varied to optimize MRA [24], to shorten total acquisition time in spectroscopic imaging [25], and to reduce truncation artifacts in chemical-shift imaging [26].

Despite all these improvements in acquisition speed, only one image of a particular contrast is generally acquired with each technique. If multiple images of various contrasts are needed (e.g., a $T_{1}$ - and a $T_{2}$-weighted image for clinical diagnosis), image acquisition must be repeated with different acquisition parameters. The resulting total scan time for all images is then the sum of the scan time for each image with a specific contrast. Moreover, often a new scan setup, such as slice prescription, is required to obtain an additional image of a different contrast of the same geometry.

Hence, to overcome the constraints of single-contrast imaging, researchers have pursued the idea of acquiring multicontrast images. Multicontrast acquisitions include multiecho 
spin echo (SE), dual-contrast gradient-spin echo (GRASE) [27], dual-echo fast fluid-attenuated inversion recovery (fFLAIR) [28], and dual-contrast FSE [29] sequences. The reconstruction of two images of different contrasts from different signal components in a steady-state gradient echo sequence was first reported in [30]. In addition, the combination of two FSE sequences (8-echo and 4-echo) with two different repetition times was suggested in [31] to obtain images of three different contrasts $\left[T_{1^{-}}\right.$, proton density (PD)-, and $T_{2}$-weighted] in one acquisition. In contrast to the work presented in this paper, sharing of $k$-space data between images of different contrasts was not proposed for these techniques, except for a dual-contrast FSE sequence [32]. On the other hand, the concept of PE view sharing was previously applied for the efficient creation of a series of images over time [33] as opposed to contrast. These studies led to the development of keyhole imaging [7], [8], and other approaches for dynamic MRI.

In this paper, a new technique of combining the acquisition of images of different contrasts into a single acquisition, using variable acquisition parameters and $k$-space data sharing is introduced. This type of acquisition is termed "combo acquisition". In contrast, conventional imaging with fixed acquisition parameters is referred to as "standard acquisition." The novelty of combo acquisitions is the systematic integration of the three concepts of: 1) variable acquisition parameters; 2) $k$-space data sharing; and 3) multicontrast imaging into the design of a single acquisition protocol. The goals of developing such an integrated technique were a) scan time reduction and b) more efficient data utilization in clinical practice. The latter goal is motivated by the observation of under-utilization of data from scans of the same object/patient (same spins) that have distinct contrast weighting. For instance, information from a $T_{1}$-weighted scan is not utilized in a $T_{2}$-weighted scan in any way. To better utilize acquired data in combo acquisitions, high-frequency data with respect to PE (large $k_{y}$ values) are shared in the reconstruction of multiple images of different contrasts. In general, most of the signal energy in the $k$-space of an MRI scan is concentrated at low spatial frequencies (small $k_{y}$ values) that determine the contrast. Hence, it is assumed that high-frequency data sharing neither strongly degrades image contrast nor significantly increases the level of artifacts. In addition to $k$-space data sharing, the paradigm of fixed acquisition parameters is replaced by (continuously) varying parameters TR and TE and echo train length (ETL) during the acquisition of all $\mathrm{PE}$ views in spin echo based combo scans. Thus, different PE views in $k$-space are acquired with distinct acquisition parameters. Signals for low frequencies are separately acquired for each image and used to preserve contrast. However, the sharing of high-frequency data leads to significant scan time reduction. Finally, it is important to note that the idea of a combo acquisition is pertinent to a general clinical setting and not only to selected applications. No specific scanner or gradient hardware is required. The method is implementable on most commercially available scanners, including those already in clinical use.

In Section II, we describe a simulation of this method to show its theoretical and practical advantages and limitations. First, simulation of MRI data acquisition using Bloch equations is de- scribed. In subsequent subsections, we elaborate on the design, optimization, and evaluation of combo acquisition protocols.

\section{METHODS}

\section{A. Simulation of MRI Data Acquisition}

Acquisition of MR signals was simulated by solving the Bloch equations [34] in the rotating frame of reference for each point of an object. Objects were generated as phantom data with object points (voxels) modeled as magnetization vectors at locations $\vec{r}=(x, y, z)^{T}$ and with physical properties, such as spin density $\rho$, gyromagnetic ratio $\gamma$, and longitudinal and transverse relaxation times $T_{1}$ and $T_{2}$, respectively.

The phenomenological Bloch equations provide a classical description of the time evolution of a magnetization vector $\vec{M}=$ $\left(M_{x}, M_{y}, M_{z}\right)^{T}$ in the presence of a magnetic field including relaxation effects for static spins (no diffusion terms)

$$
\frac{d \vec{M}}{d t}=\gamma \vec{M} \times \vec{B}_{\text {ext }}+\frac{1}{T_{1}}\left(M_{0}-M_{z}\right) \hat{z}-\frac{1}{T_{2}} \vec{M}_{\perp}
$$

where, $\gamma=42.576 \mathrm{MHz} / \mathrm{T}$ for protons, $\vec{B}_{\text {ext }}$ is an external magnetic field, $M_{0}$ is the equilibrium magnetization of the system, $M_{z}$ is the magnetization along the $z$ axis, and $\vec{M}_{\perp}$ is the magnetization vector in the transverse $(x-y)$ plane. In this paper, the Bloch equations were solved for each object point of phantom data to compute the resulting MR signal for the events of spin echo (SE) and FSE sequences using the Matlab programming language (The MathWorks Inc., Natick, MA). Signal contributions were not computed from expressions for the signal magnitude for fixed acquisition parameters, since a steady-state magnetization is usually assumed for these expressions. Steady state is not guaranteed when using variable TR. Following simulated data acquisition, images were reconstructed using an inverse fast Fourier transform (IFFT) of sampled $k$-space data. In combo acquisitions, signal matrices were reordered for each image prior to reconstruction. Phenomena, such as diffusion, flow, chemical shift (water/fat separation), main field inhomogeneities $\Delta B(\vec{r})$ (leading to $T_{2}^{*}$ - decay), radiofrequency (RF) - inhomogeneities, and magnetization transfer were not simulated.

For all experiments, the realistic brain phantom model from the McConnell Brain Imaging Centre (BIC) of McGill University [35], [36] was used. This 3-D digital brain phantom generated from real MRI data consists of ten volumetric data sets that define the spatial distribution for different tissues, e.g., white matter (WM), gray matter (GM), cerebrospinal fluid (CSF), muscle, skin, etc.. A voxel could have multiple tissue contributions, i.e., magnetization vectors with the same spatial coordinates, but different MRI relevant parameters such as $T_{1}, T_{2}$, and $\rho$. Here, differences introduced by various tissue types refer to relaxation parameters and spin density, not to a distribution change in Lamor frequencies of the different components. For the purposes of this study, volumes of different tissues that are most dominant for MR imaging of the brain, such as WM, GM, and CSF, were selected. In addition, tissue with pathology [multiple sclerosis (MS) lesions] was chosen to investigate lesion conspicuity with combo acquisitions. 
TABLE I

PHYSICAL PARAMETERS OF TISSUES WM, GM, AND CSF

\begin{tabular}{c|c|c|c}
\hline Tissue & $\mathbf{T}_{\mathbf{1}}(\mathbf{m s})$ & $\mathbf{T}_{\mathbf{2}}(\mathbf{m s})$ & $\boldsymbol{\rho}\left(\mathbf{1 0}^{\mathbf{3 1}} \mathbf{\mathbf { c m } ^ { \mathbf { 3 } } )}\right.$ \\
\hline \hline WM & 600 & 80 & 0.61 \\
\hline GM & 950 & 100 & 0.69 \\
\hline CSF & 4500 & 2200 & 1.0 \\
\hline
\end{tabular}

Relaxation times and spin density of selected tissues for a main field $B_{0}=1.5$ tesla $(\mathrm{T})$ were obtained from the literature [37] and are summarized in Table I. Phantom data of in-plane $(x, y)$-resolutions of $0.1 \mathrm{~cm} \times 0.1 \mathrm{~cm}$ were generated. For simplicity, it was assumed that phantom models were uniform along the $z$ direction for the entire selected slice thickness. For simulated MRI signal acquisitions, a square $\mathrm{FOV}_{\text {in-plane }}=$ $25.6 \mathrm{~cm}, N_{x}=256$ frequency encoding steps, $N_{y}=256 \mathrm{PE}$ views, and number of averages NEX $=1$ were chosen. Magnitude images were reconstructed at size of $256 \times 256$ pixels. Note that in the context of simulated data acquisition we use the term "predicted efficiency in scan time" or simply "predicted scan time" instead of "scan time," since the latter implies experimental data acquisition on a real MR scanner.

\section{B. Design Considerations for Combo Acquisition Protocols}

The design of combo acquisition protocols can be described using the following three major criteria:

1) amount and distribution of $k$-space data sharing;

2) variation schemes for selected acquisition parameters;

3) exact ordering of all PE views.

To choose the amount and distribution (spatial frequency content) of $k$-space data sharing between images of different contrasts in combo acquisitions, PE schemes were first developed. For this, the total number of $N_{y}=256 \mathrm{PE}$ views was divided into segments of 8, 16, 32, or 64 for each image. Data for a specific segment was either separately generated for each image of different contrast, or shared in the reconstruction of multiple images. The total number of all PE segments for a given number of contrasts was then related to the amount of data sharing. The distribution of shared and separately generated segments was based on their respective spatial frequency content with the general rule that signals for low-frequency PE views were separately synthesized, whereas high-frequency data was often shared. An example of such a scheme for a 2-contrast SE combo acquisition is shown in Fig. 1 with a total number of six segments. Each segment contained $64 \mathrm{PE}$ views. The first four segments were used to reconstruct a $T_{1}$-weighted image. A $T_{2}$-weighted image was reconstructed from the last four segments. This is also indicated in Fig. 1. Stepping through all PE numbers was carried out in zigzag fashion, i.e., $N_{\mathrm{PE}}=$ $\ldots, 0,-1,1,-2,2, \ldots,\left(+N_{y} / 2-1\right),\left(-N_{y} / 2\right)$.

To obtain different contrast weightings in combo acquisitions and to improve the predicted efficiency in scan time of combo acquisitions, variation schemes were designed for selected scan parameters. In SE combo acquisitions, the acquisition parameters TR and TE were varied. This concept was extended in FSE combo acquisitions by varying parameters TR, echo spacing (ESP), and ETL. Variation of the parameter ESP in FSE se-

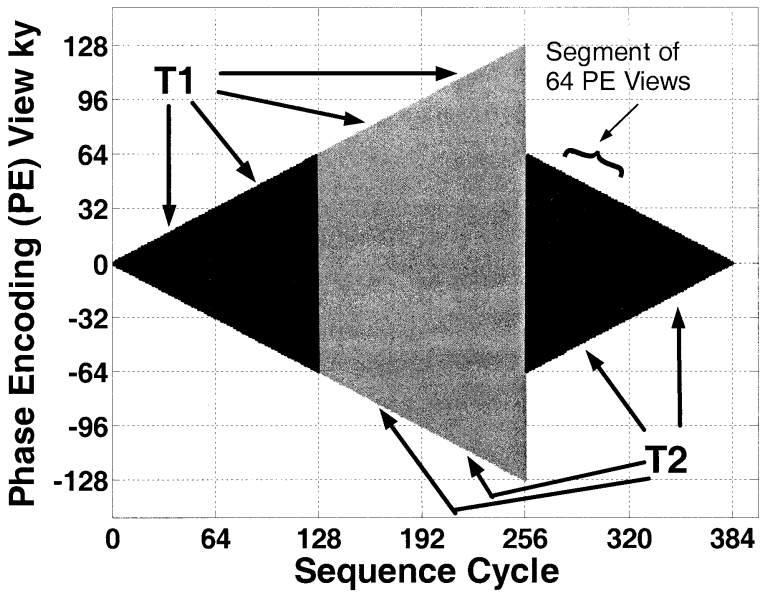

Fig. 1. PE scheme for a 2-contrast SE combo acquisition $\left(T_{1}-T_{2}\right)$ with 384 sequence cycles. Arrows indicate segments of PE views used for reconstruction of a $T_{1}$ - and a $T_{2}$-weighted image, respectively.

quences essentially corresponds to the use of variable TE in SE imaging. More precisely, ESP is the fixed time interval between different echoes and it defines the timing for all echoes in a FSE echo train by $\mathrm{TE}_{p}=p \cdot \mathrm{ESP}$, where $p$ is the number of an echo and $p=1,2, \ldots$, ETL. The effective echo time $\mathrm{TE}_{\mathrm{eff}}$ for a FSE sequence is then determined by the echo time, at which the zero PE view $\left(k_{y}=0\right)$ for an image is acquired [38]. In general, the range of parameter variation strongly depended on the choice of different contrasts to be obtained in a multicontrast combo scan. For example, to obtain images of all three contrasts in a SE combo acquisition, TR was varied from $500 \mathrm{~ms}$ to $2500 \mathrm{~ms}$, and TE from 20 to $150 \mathrm{~ms}$. However, variation of acquisition parameters did not necessarily have to be continuous to preserve contrast and to yield images with few artifacts. Moreover, since combo acquisitions are intended for scan time reduction in a clinical setting, parameter variation schemes had to be designed to accommodate multislice imaging. For 3-contrast combo acquisitions the minimum number of slices for which data can be generated in each sequence cycle was set to $N_{\text {slices }}=15$. For the design of $T_{1}-T_{2} \mathrm{SE}$ combo acquisitions, this requirement had not been considered, but could be imposed in future schemes. The constraint of multislice imaging limited the range for parameter TE for a given TR in SE combo scans and for parameter ETL for given TR and ESP in FSE combo acquisitions. For instance, with the requirement of 15 slices for a FSE combo acquisition, and with the choice of $\mathrm{ESP}=15 \mathrm{~ms}$ and $\mathrm{TR}=500 \mathrm{~ms}$, ETL could not exceed 2.

Finally, the exact ordering of all $P E$ views within the segments of a PE scheme was determined for a particular set of parameter variation curves of a combo acquisition. Due to the multiecho structure of the FSE sequence and its corresponding nonuniform $k$-space data weighting along the PE direction [39], PE ordering gained increased importance to reduce resulting image artifacts in FSE combo acquisitions.

\section{Optimization and Evaluation of Combo Acquisition Protocols}

After an initial design of a combo acquisition, our goal was to optimize the protocol by preserving desired contrast 


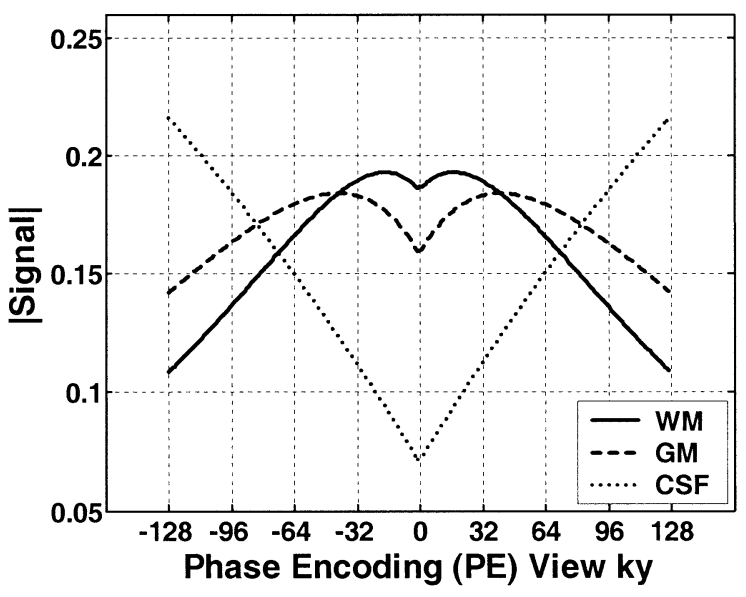

(a)

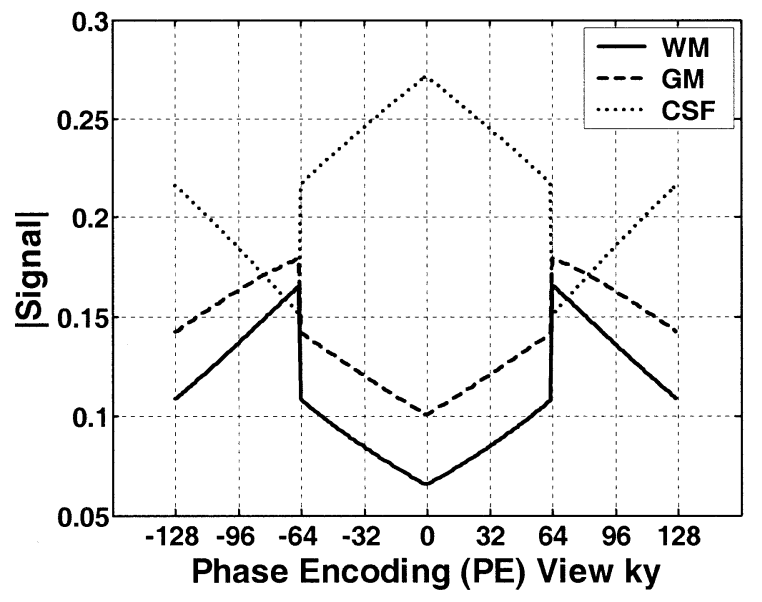

(b)

Fig. 2. Signal levels according to linear variation of TR and TE for tissues WM, GM, and CSF for (a) a $T_{1^{-}}$, and (b) a $T_{2}$-weighted image of a 2 -contrast SE combo acquisition.

and minimizing visible artifacts in the resulting images. In combo acquisitions, artifacts in the image domain are inevitably introduced through nonuniform data weighting in $k$-space. Nonuniform and tissue dependent data weighting in $k$-space, in turn is caused by varying selected acquisition parameters over all PE views of a particular image, similar to data acquisition with multiple echoes after a single RF-excitation, e.g., using a conventional FSE sequence [2]. In general, the optimization process was semi-empirical, tissue dependent, and was carried out in an iterative fashion. In addition to preservation of contrast, the optimization procedure was guided by analyzing signal levels (data weighting in $k$-space) and minimizing three quantitative criteria: the energy of the "residue point spread function" (PSF) $E\left(\mathrm{PSF}_{\mathrm{res}}\right)$, the energy of "residue profiles" across sharp tissue boundaries $E$ (profiles res ), and the energy of "residue images" $E$ (images $_{\text {res }}$ ). During optimization, results from combo acquisitions were compared with images from standard acquisitions with fixed acquisition parameters in terms of image contrast, level of artifacts, and resolution. The final results were further evaluated by quantitatively analyzing the preservation of contrast, the point spread function (PSF), and the signal-to-noise ratio (SNR) for each image.

Next, we describe the optimization procedures in more detail.

1) Preservation of Contrast: To preserve image contrast, the signal for the zero PE view $\left(k_{y}=0\right)$ for each image in a combined acquisition was computed with specific settings for selected acquisition parameters, such as TR and TE. These settings were the same as those used to obtain a specific contrast in a corresponding standard acquisition. In general, data for lowfrequency PE views with $\left|k_{y}\right|<\left(\left|k_{y, \max }\right| / 2\right)$ were generated with values close to these settings for contrast preservation. To shift from one contrast weighting to another, acquisition parameters were more rapidly varied during the acquisition of high-frequency PE views with $\left|k_{y}\right|>\left(\left|k_{y, \max }\right| / 2\right)$. These $\mathrm{PE}$ views contribute less to the contrast, but carry significant and subtle details of an image. Contrast measurements were performed to evaluate the preservation of contrast with combo acquisitions.
2) Analysis of Signal Levels (K-Space Data Weighting): For semi-empirical optimization of combo acquisitions signal levels (magnitudes) for a single object point of each tissue were generated. For this, data was separately synthesized with PE set to zero using selected parameter variation schemes. Signal levels correspond to data weighting in $k$-space with respect to PE views $\left.k_{y}\right|_{k_{x}=0}$. An example for the signal levels for a 2-contrast $\mathrm{SE}$ combo acquisition with linear variation of both, TR and TE, is shown in Fig. 2. The set of signal levels of all tissues for each image according to a selected parameter variation scheme was then analyzed. Variation curves for acquisition parameters were modified, where possible, to preserve the order of signal levels for a particular contrast, e.g., $\mid$ signal $_{\mathrm{WM}}|>|$ signal $_{\mathrm{GM}}|>|$ signal $_{\mathrm{CSF}} \mid$ for a $T_{1}$-weighted image, and to minimize discontinuities between signal levels for different segments of PE views.

3) Quantitative Optimization Criteria: An iteration of the optimization process for combo acquisition protocols corresponded to one or more modifications of PE and/or parameter variation schemes. Results obtained with the modified protocol were assessed by computing three quantitative criteria: the energy of the "residue PSF" $E$ ( $\mathrm{PSF}_{\text {res }}$ ), the energy of "residue profiles" across sharp tissue boundaries $E$ (profiles $_{\text {res }}$ ), and the energy of "residue images" $E$ (images (imes ). In essence, each criterion measured the total energy of the squared absolute difference of a selected characteristic, such as the PSF, between combo and corresponding standard acquisitions. Optimization of combo acquisition was achieved by minimizing all three quantitative criteria, since minimization of these criteria corresponded to a reduction of artifacts. Modifications of PE and variation schemes to optimize combo acquisitions were therefore kept or rejected based on their effect on these criteria. The mathematical definition of the three quantitative criteria and the description of all evaluation steps are presented later in Section III-E together with numerical results.

In summary, the initial design of a combo acquisition was optimized to preserve contrast and minimize artifacts. Each iteration of the optimization process was assessed using signal levels 
TABLE II

ACQUiSITION PARAMETERS FOR STANDARD ACQUISITIONS OF THREE DIFFERENT CONTRASTS FOR SE (TR AND TE) AND FSE (ETL, ESP, TR, AND TE eff SEQUENCES) (NEX $=1$ ) USED AS REFERENCES For COMBO ACQUISITIONS

\begin{tabular}{l|c|c|c|c|c|c}
\hline \multicolumn{3}{c|}{ Standard Acquisitions for SE Sequences } & \multicolumn{4}{c}{ Standard Acquisitions for FSE Sequences } \\
\hline \hline Contrast & TR $(\mathbf{m s})$ & TE $(\mathbf{m s})$ & ETL & ESP $(\mathbf{m s})$ & TR (ms) & TE $_{\text {eff }}\left(\mathbf{m s}_{\mathbf{n}}\right)$ \\
\hline $\mathrm{T}_{1}$ & 500 & 20 & 1 & 15 & 500 & 15 \\
\hline $\mathrm{PD}$ & 2500 & 20 & 8 & 20 & 2500 & 20 \\
\hline $\mathrm{T}_{2}$ & 2500 & 150 & 8 & 20 & 2500 & 140 \\
\hline
\end{tabular}

and three quantitative criteria. Final results were quantitatively evaluated and are described next.

\section{RESULTS}

Results for 2-contrast and 3-contrast SE and FSE combo acquisitions are presented. Reconstructed images are either $T_{1^{-}}$, PD-, or $T_{2}$-weighted. These images were compared with images from corresponding standard acquisitions. The latter served as references in terms of image contrast, quality, and resolution. Images shown are simulated magnitude images.

\section{A. Images From Standard Acquisition Protocols}

As standard acquisitions for SE combo acquisitions, image $k$-space results of the three different contrasts $\left(T_{1-}, \mathrm{PD}-\right.$, and $T_{2}$-weighted) were computed using a single TR and a single TE. The choices of acquisition parameters TR and TE for these contrasts are summarized in Table II (NEX is assumed to be one). For 3-contrast FSE combo acquisitions, a combination of a SE sequence for the $T_{1}$-weighted image and a dual-contrast FSE sequence with no data sharing for the other two images was selected as standard acquisitions. Parameters for these two scans are also included in Table II. Note that the predicted scan time reduction obtained with combo acquisitions depends on the choice of the corresponding standard acquisitions used as a reference.

\section{B. Images From SE Combo Acquisition Protocols}

1) Images From $T_{1}-T_{2} S E$ Combo Acquisition Protocols: Initial experiments focused on a combined acquisition of a $T_{1}$ - and a $T_{2}$-weighted image using a $\mathrm{SE}$ sequence, since these two contrasts are of greatest importance in clinical diagnosis. Using the PE scheme shown in Fig. 1, TR and TE were varied linearly over all $\mathrm{PE}$ views from minimum values, $\mathrm{TR}_{\min }=500 \mathrm{~ms}$ and $\mathrm{TE}_{\min }=20 \mathrm{~ms}$, to maximum values $\mathrm{TR}_{\max }=2500 \mathrm{~ms}$ and $\mathrm{TE}_{\max }=150 \mathrm{~ms}$, respectively. Linear variation of TR and TE was initially chosen to obtain a smooth shift in data weighting from one contrast to another and had been used by other researchers before [21]. The corresponding results are shown in Fig. 3. As it can be seen from Fig. 3, both images exhibit severe artifacts. The $T_{1}$-weighted image in Fig. 3(a) shows brightening of CSF, especially at the edges of the brain area [see arrow in Fig. 3(a)], some amount of blurring, and ringing artifacts, which are worst for areas of CSF content. Ringing can also be observed in the $T_{2}$-weighted image in Fig. 3(b), where it is most severe for WM [see arrow in Fig. 3(b)]. Fine structures are blurred. Image contrast is preserved in both images, except for the brighter $\mathrm{CSF}$ in the $T_{1}$-weighted image already mentioned. Artifacts

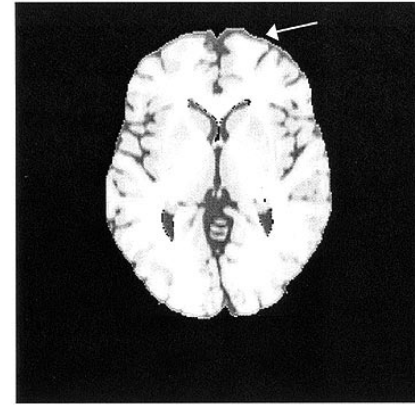

(a)

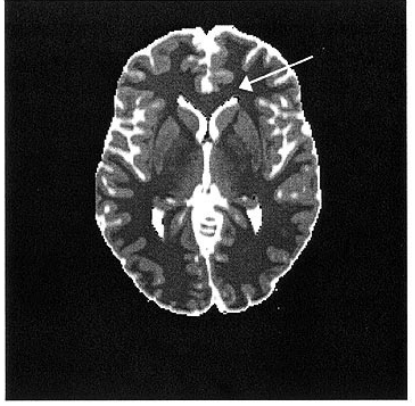

(b)
Fig. 3. Simulated images from a 2-contrast SE combo acquisition with linear variation of TR and TE: (a) $T_{1}$ - and (b) $T_{2}$-weighted image. Predicted scan time was reduced by $25 \%$. Arrows indicate image artifacts: (a) brightening of CSF at the edges of the brain area and (b) ringing in the WM caudal to the corpus callosum.

can be examined using the curves for the corresponding signal levels in Fig. 2 and by considering the relaxation times of the different tissues. By increasing TE linearly, signal levels for WM and GM decreased quickly, whereas the signal level for CSF were less sensitive to changes of TE due to its longer $T_{2}$ relaxation time. In contrast, the signal level for CSF increased with increasing TR, so that it showed the highest value of the three tissues for PE numbers with $\left|k_{y}\right|>80$ as can be seen in Fig. 2(a). Hence, brightening of CSF and ringing in the $T_{1}$-weighted image are seen in Fig. 3(a). As shown in Fig. 2(b), signal levels for WM and GM were elevated for PE views with $\left|k_{y}\right|>64$ compared with PE views with $\left|k_{y}\right|<64$ leading to ringing artifacts in the $T_{2}$-weighted image in Fig. 3(b). These observations suggested that TR should increase more slowly during the generation of $k$-space data for the $T_{1}$-weighted image to prevent a high signal level for CSF, thus to prevent the brightening of CSF. Similarly, TE should be changed less aggressively for low-frequency $\mathrm{PE}$ views of the $T_{1}$-weighted image to keep signal levels for WM and GM at higher values. In contrast, TE should be varied more rapidly during the simulated acquisition of high-frequency $\mathrm{PE}$ views before it should again vary only slowly for the low-frequency PE views of the $T_{2}$-weighted image.

To follow these guidelines, several sigmoidal and sinusoidal functions were used to vary TR and TE. Here, sigmoidal variation curves $f_{\text {sigm }}$ were expressed as

$$
\begin{aligned}
& f_{\operatorname{sigm}}(s)=c_{1}+c_{2} \cdot \operatorname{sigm}(s) \\
& \operatorname{sigm}(s)=\left(1+e^{-s}\right)^{-1}
\end{aligned}
$$

with $s \in[a, b]$. Parameters $c_{1}$ and $c_{2}$ are constants depending on the minimum and maximum values of the desired variation. 


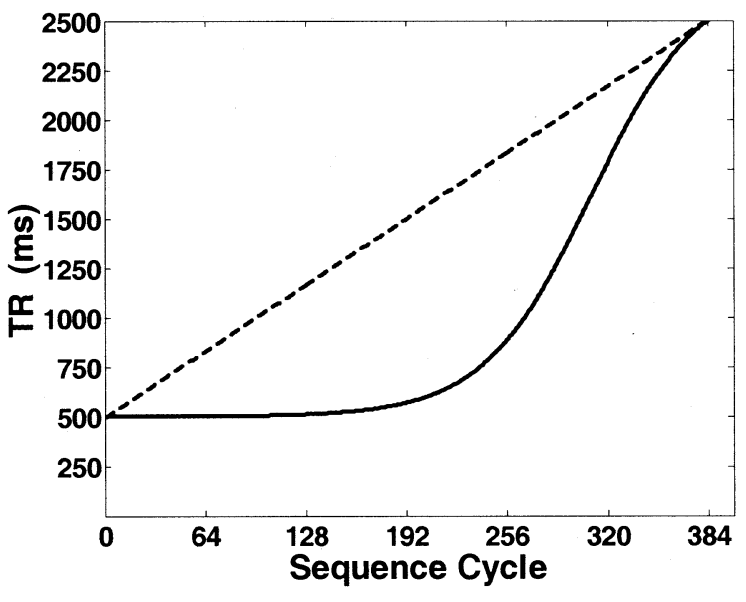

(a)

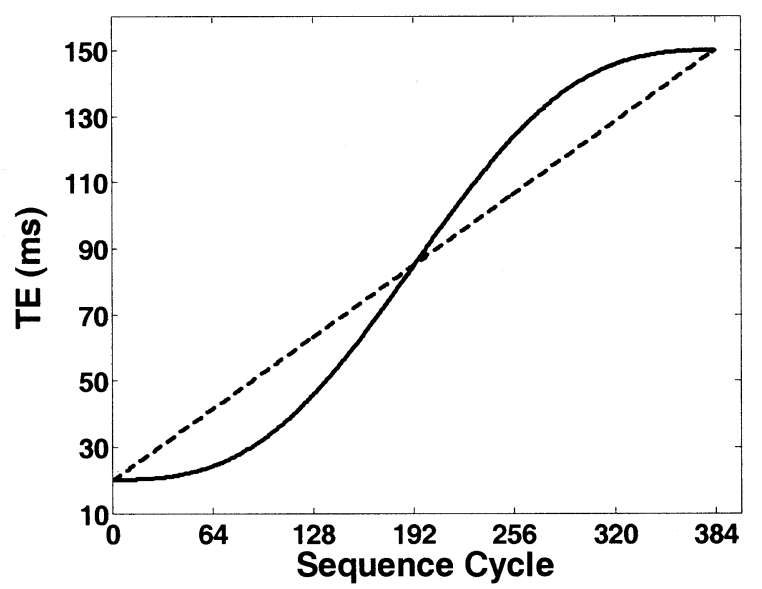

(b)

Fig. 4. Optimized parameter variation scheme for a 2-contrast SE combo acquisition $\left(T_{1}-T_{2}\right)$ with 384 sequence cycles: (a) sigmoidal curve for TR and (b) sinusoidal curve for TE as solid lines. Linear curves for TR and TE are shown as dashed lines for comparison.

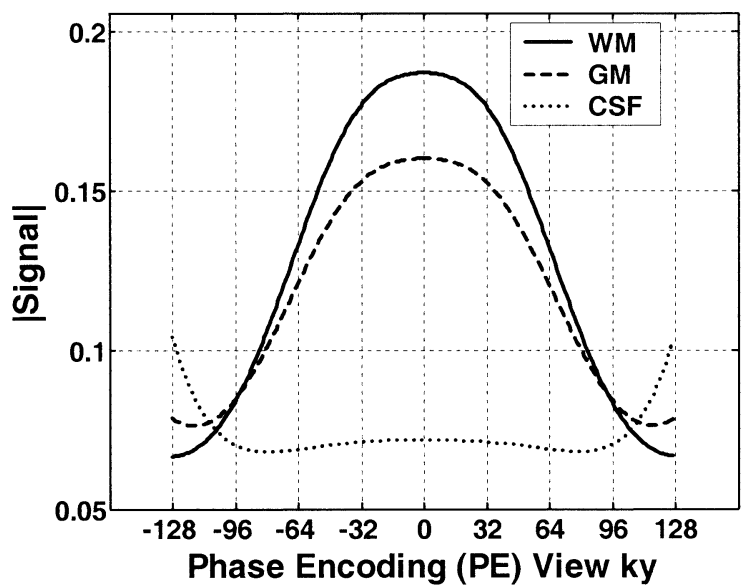

(a)

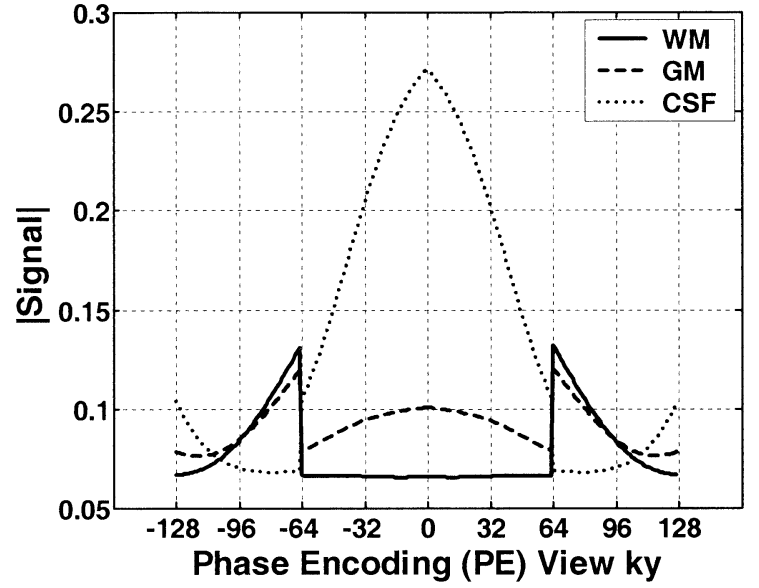

(b)

Fig. 5. Signal levels according to the variation scheme of a 2-contrast SE combo acquisition in Fig. 4 for tissues WM, GM, and CSF for (a) a $T_{1-}{ }^{-}$, and (b) a $T_{2}$-weighted image.

Changing these constants and the interval $[a, b]$ yields different sigmoidal functions. One choice for a variation scheme that produced results without major artifacts is shown in Fig. 4. Parameters for the curve for TR in Fig. 4(a) were chosen as follows:

$$
\begin{aligned}
c_{1} & =\mathrm{TR}_{\max }-\frac{\left(\mathrm{TR}_{\max }-\mathrm{TR}_{\min }\right) \cdot \operatorname{sigm}(b)}{(\operatorname{sigm}(b)-\operatorname{sigm}(a))} \\
c_{2} & =\frac{\left(\mathrm{TR}_{\max }-\mathrm{TR}_{\min }\right)}{(\operatorname{sigm}(b)-\operatorname{sigm}(a))} \\
a & =-9, b=\ln (9)
\end{aligned}
$$

where, values for $a$ and $b$ were obtained empirically. For the sinusoidal curve for TE in Fig. 4(b) the analytical formulation is

$$
\begin{aligned}
& f_{\sin }(s)=\mathrm{TE}_{\min } \\
& \quad+\frac{\mathrm{TE}_{\max }-\mathrm{TE}_{\min }}{\left(N_{\text {cycles }}-1\right)} \cdot s-20 \cdot \sin \left(s \cdot \frac{2 \pi}{\left(N_{\text {cycles }}-1\right)}\right)
\end{aligned}
$$

where $s \in\left[0,\left(N_{\text {cycles }}-1\right)\right]$ and $N_{\text {cycles }}=384$ is the total number of sequence cycles. Signal levels corresponding to the variation scheme in Fig. 4 are presented in Fig. 5, and the resulting images are shown in Fig. 6. Contrast was preserved in both images as can be seen by comparing the images in Fig. 6(a) and (c) with the corresponding images from standard acquisitions shown in Fig. 6(b) and (d), respectively. The $T_{1}$-weighted image in Fig. 6(a) shows only minor brightening of CSF and nearly no ringing artifacts, though the $T_{2}$-weighted image in Fig. 6(c) shows some minor ringing within the areas of WM and GM. Fine structures of tissues WM and GM though were somewhat blurred in the $T_{1}$-weighted image due to relatively small signal levels for PE views with $|k y|>96$ as seen in Fig. 5(a) that resulted in a widened PSF. A similar observation holds for CSF features in the $T_{2}$-weighted image. On the other hand, decreased signal levels for CSF in Fig. 5(a) compared with Fig. 2(a) and for WM and GM in Fig. 5(b) compared with Fig. 2(b) entailed in a reduction of artifacts seen in the images in Fig. 3. For this specific 2-contrast SE combo acquisition, 


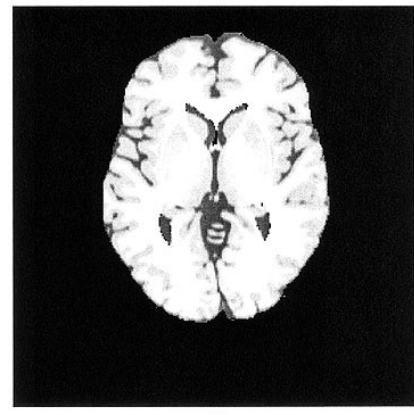

(a)

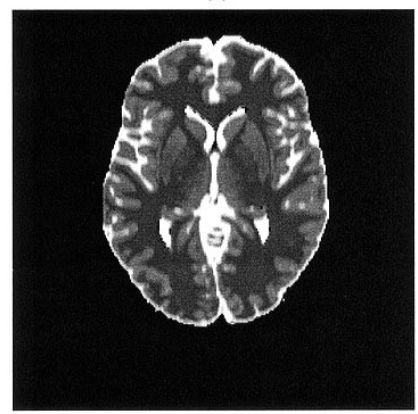

(c)

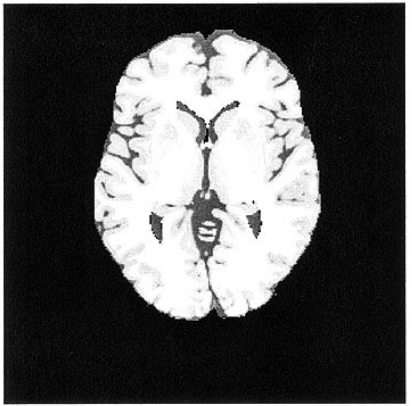

(b)

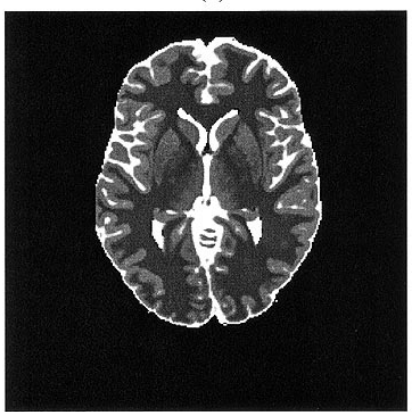

(d)
Fig. 6. Simulated images for two different contrasts, $T_{1^{-}}$, and $T_{2}$-weighted: Images in (a) and (c) from a 2-contrast SE combo acquisition using the variation scheme in Fig. 4, and images in (b) and (d) from corresponding standard acquisitions with parameters from Table II. Predicted scan time was reduced by $52 \%$ with the combo acquisition.

a predicted scan time reduction of $52 \%$ was achieved relative to the predicted scan time needed for two separate standard acquisitions. A similar scheme that used sigmoidal functions to vary both, TR and TE, produced a $47 \%$ predicted scan time reduction. In this case, the $T_{1}$-weighted image was slightly more blurred, but the $T_{2}$-weighted image was improved showing even less ringing artifacts. This observation illustrates a trade-off between the optimization of one contrast weighting at the cost of another.

2) Images From 3-Contrast SE Combo Acquisition Protocols: The concept of SE combo acquisitions was also applied to the acquisition of three different contrasts. As before, optimization of acquisition protocols was carried out semi-empirically and iteratively. A set of specific variation curves for TR and TE resulting from these experiments is shown in Fig. 7(a). The corresponding PE scheme is shown in Fig. 7(b). Arrows in Fig. 7(b) indicate segments of PE views that were used to reconstruct a $T_{1^{-}}$, PD- and a $T_{2}$-weighted image, respectively. Reconstructed images are presented in Fig. 8 together with matching images from standard acquisitions. As seen in Fig. 8, images from the SE combo acquisition have sufficient contrast definition and do not exhibit significant artifacts. In particular, the $T_{1}$ - and the $T_{2}$-weighted images in Fig. 8(a) and (e), respectively, are very similar to their counterparts from standard acquisitions in Fig. 8(b) and (f). For the images shown in Fig. 8 predicted scan time was reduced by $31 \%$ compared with standard acquisitions. Signal levels at each PE view for this 3-contrast SE combo acquisition are presented in Fig. 9. As can be seen from Fig. 9(a) and (c), the order of signal levels for the corresponding contrasts was preserved for almost all PE numbers of the $T_{1}$ - and the $T_{2}$-weighted images.

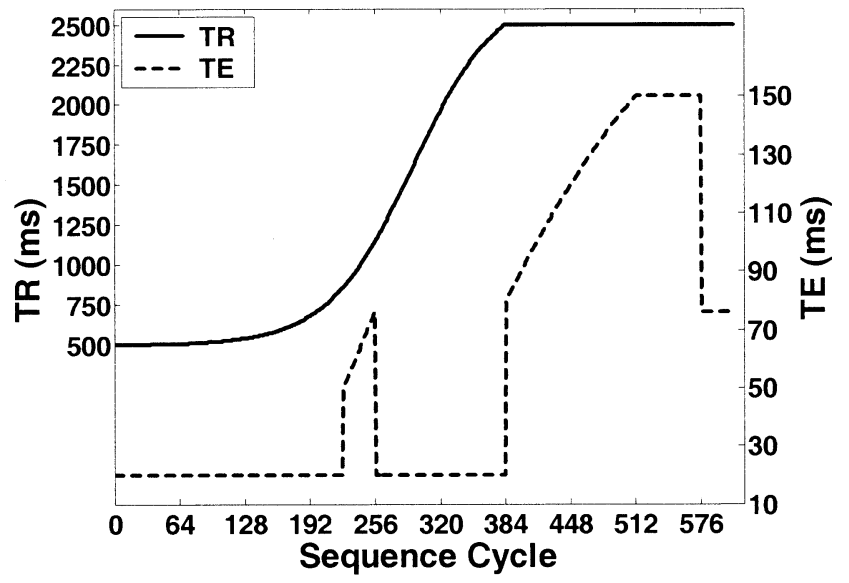

(a)

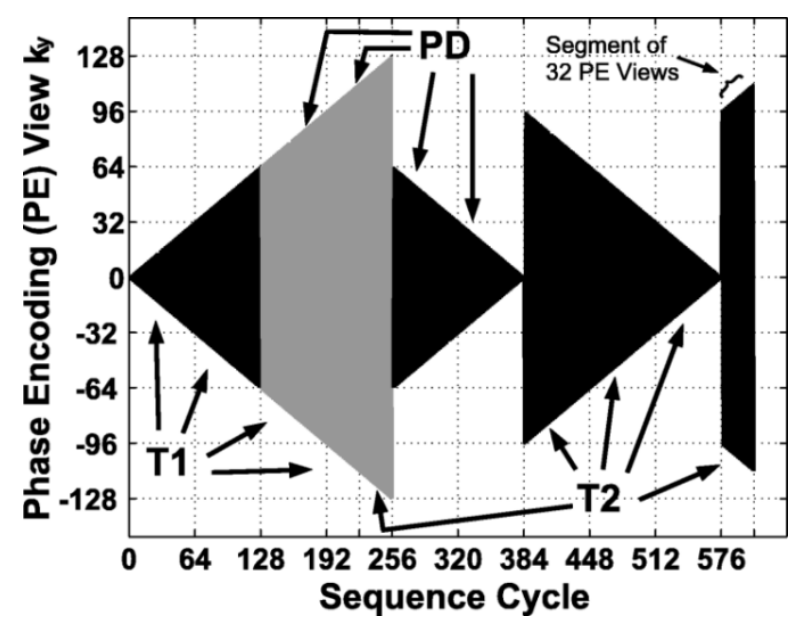

(b)

Fig. 7. Optimized parameter variation and PE schemes for a 3-contrast SE combo acquisition ( $T_{1}, \mathrm{PD}$, and $\left.T_{2}\right)$ with 608 sequence cycles. (a) Curves of variation for TR (solid) and TE (dashed). (b) PE scheme. In (b), arrows indicate segments of PE views used for reconstruction of a $T_{1^{-}}, \mathrm{PD}$-, and $T_{2}$-weighted image, respectively.

Only signal levels for the highest frequencies were attenuated, when compared with the corresponding standard acquisitions. This explains the similarities between the images from combo and standard acquisitions. For the PD-weighted image, signal levels of higher frequencies $\left(\left|k_{y}\right|>64\right)$ were more strongly attenuated, and their order for the three tissues was modified from the one for PD-contrast as shown in Fig. 9(b). Thus, the PD-weighted image in Fig. 9(c) exhibits a slightly different visual appearance than its counterpart from a standard scan in Fig. 8(d).

3) Filtering: To correct for different data weighting in $k$-space, various filters derived from the distribution of signal levels over all PE views for the tissues WM, GM, and CSF according to a selected variation scheme were applied. In general, filtering had limited benefits: some artifacts were removed, while others were amplified. Optimal filtering is at an impasse in this case, since contributions from different tissues from all voxels can no longer be separated within the synthesized signal for a particular PE view. Therefore, the effect of naïve filtering represented enhancement of the signal for one particular tissue, but diminished the signal of other tissue types. 


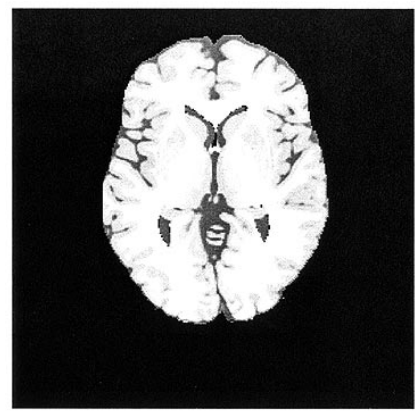

(a)

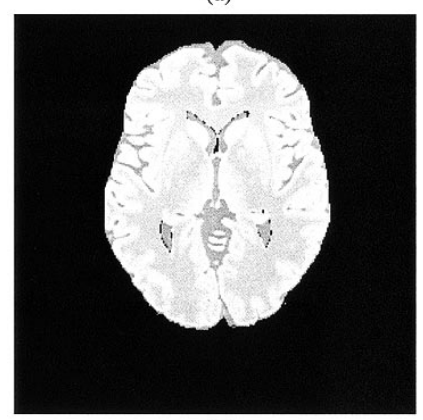

(c)

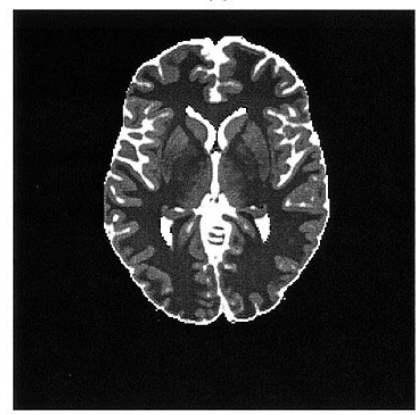

(e)

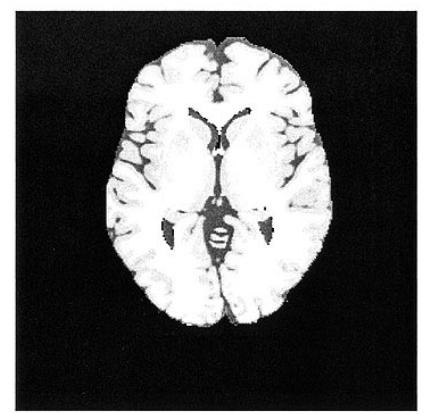

(b)

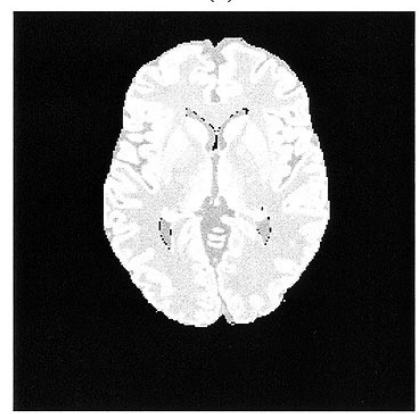

(d)

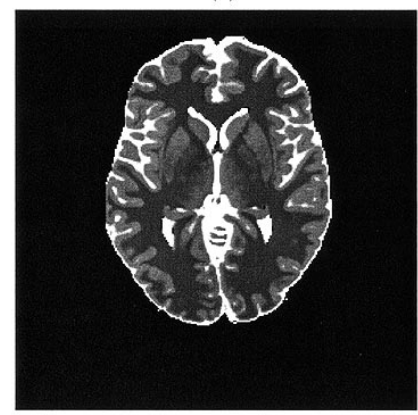

(f)
Fig. 8. Simulated images for three different contrasts, $T_{1^{-}}$, PD-, and $T_{2}$-weighted: Images in (a), (c), and (e) from a 3-contrast SE combo acquisition using the variation scheme in Fig. 7, and images in (b), (d), and (f) from standard acquisitions with parameters from Table II. Predicted scan time was reduced by $31 \%$ with the combo acquisition.

\section{Images From 3-Contrast FSE Combo Acquisition Protocols}

Combo acquisitions using the FSE sequence and achieving scan time reduction would make this technique more practical and more useful. Hence, simulations were extended to include the application of the FSE sequence in multicontrast acquisitions. As with SE combo scans, initial acquisition protocols for 3-contrast FSE combo acquisitions were refined and optimized based on experimental outcome and design principles described in Section II. Semi-empirically optimized variation schemes for parameters TR (top, solid), ESP (top, dashed), and ETL (bottom, solid) are presented in Fig. 10(a). Data sharing between images is illustrated through parentheses that essentially comprise the particular set of MR signals used to reconstruct a $T_{1}-$, PD-, or a $T_{2}$-weighted image, respectively. As seen in Fig. 10(a), data for the $T_{1}$-weighted image was mainly generated with short $\mathrm{TR}$ and short $\mathrm{TE}_{p}$ (i.e., from early echoes). Signals from mainly early echoes and longer TR, were assigned to the PD-weighted image, whereas data from later echoes, i.e., long $\mathrm{TE}_{p}$, and long $\mathrm{TR}$, were used for the reconstruction of the image with $T_{2}$-contrast. Note that parameter ESP was in- creased for the last sequence cycles of the FSE combo acquisition to obtain increased $T_{2}$-contrast for the low spatial frequencies of the $T_{2}$-weighted image. The corresponding PE scheme for this acquisition is shown in Fig. 10(b) by marking the PE views in $k$-space that are generated with each echo for sets of 32 sequence cycles. An inset in the upper left corner of Fig. 10(b) shows an enlarged $k$-space diagram for one echo. Reconstructed images are displayed in Fig. 11 together with images from standard acquisitions used as reference in the computation of the predicted scan time reduction achieved with the FSE combo acquisition. Signal levels ( $k$-space data weighting) for this 3-contrast FSE combo acquisition are presented in Fig. 12. As can be seen in Fig. 11, image contrast is well preserved, and artifacts have been significantly suppressed through semi-empirical optimization of the acquisition protocol. Similar to the results of the 3-contrast SE combo acquisition in Fig. 8, the $T_{1}$ - and the $T_{2}$-weighted images in Fig. 11(a) and (e) closely resemble their counterparts from standard acquisitions in Fig. 11(b) and (f), respectively. Minor remaining artifacts in the $T_{1}$-weighted image in Fig. 11(a), such as faint ghosting in the WM caudal to the corpus callosum, were caused by nonuniform data weighting arising from data acquisition with variable ETL $>1$ and variable TR [see Fig. 12(a)] (TR had to be increased to $680 \mathrm{~ms}$ for sequence cycles 33-96 with ETL $=3$, in order to ensure multislice imaging with 15 slices). The high-quality visual appearance of the $T_{2}$-weighted image in Fig. 11(c) is the consequence of the data weighting seen in Fig. 12(c), which is very similar to the data weighting for a conventional FSE scan with ETL $=8$ and $\mathrm{TE}_{\text {eff }}=7 \cdot \mathrm{ESP}$. Sufficient $T_{2}$-contrast in the $T_{2}$-weighted image of the combo acquisition was attained by increasing ESP for the last 20 sequence cycles. Nonuniform and nonmonotonic signal levels for the PD-weighted image shown in Fig. 12(b), especially for tissues WM and GM for $k y<(-80)$, resulted in some blurring and smearing of fine details. Similar artifacts though are also seen in the PD-weighted image in Fig. 11(d). From these observations it follows that the corresponding artifacts are largely caused by the nonuniform data weighting due to $T_{2}$-decay inherent to FSE sequences with ETL $>1$. The additional modulation of signal levels induced by varying ETL and TR do not have a large impact in this case. For the images of a 3-contrast FSE combo acquisition shown in Fig. 11 predicted scan time was reduced by $30 \%$ when compared with a combination of a SE sequence for the $T_{1}$-weighted image and a dual-contrast FSE sequence with no data sharing for the other two images. If one-fourth of the acquired PE views was shared between the two images of the dual-contrast FSE sequence [32], predicted scan time was reduced by $25 \%$. Table III summarizes predicted scan time reductions for the SE and FSE combo acquisitions presented and for additional combinations not shown. It should be noted that the precision of the values in Table III refers to controlled simulation studies. In practice, these numbers will vary with the amount of $k$-space data sharing and the choice of other sequence parameters, such as TR and NEX, actually used for MR data acquisition.

\section{Multislice Images}

$T_{1}$-weighted images of six axial slices of phantom data with tissues WM, GM, and CSF and generated with the 3-contrast FSE combo acquisition protocol shown in Fig. 10, are presented 


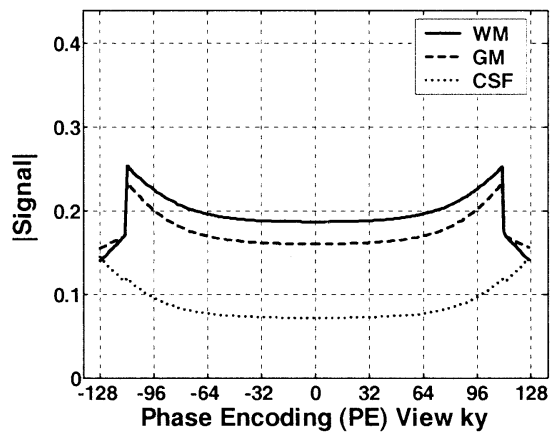

(a)

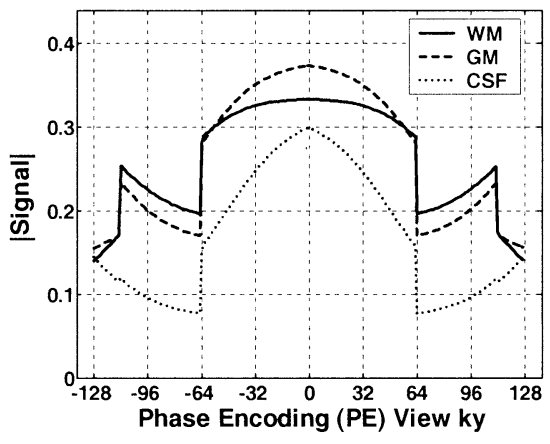

(b)

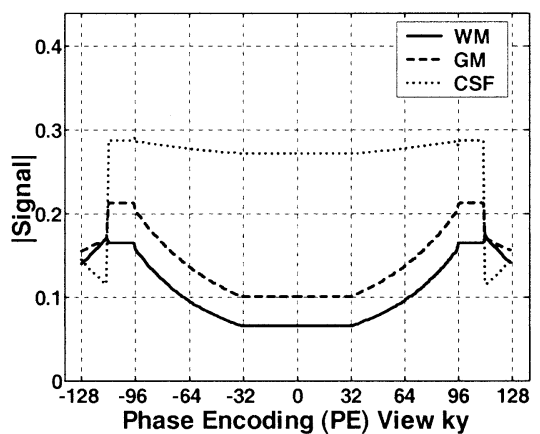

(c)

Fig. 9. Signal levels for tissues WM, GM, and CSF for (a) a $T_{1^{-}}$, (b) a PD-, and (c) a $T_{2}$-weighted image of the 3-contrast SE combo acquisition in Fig. 8.

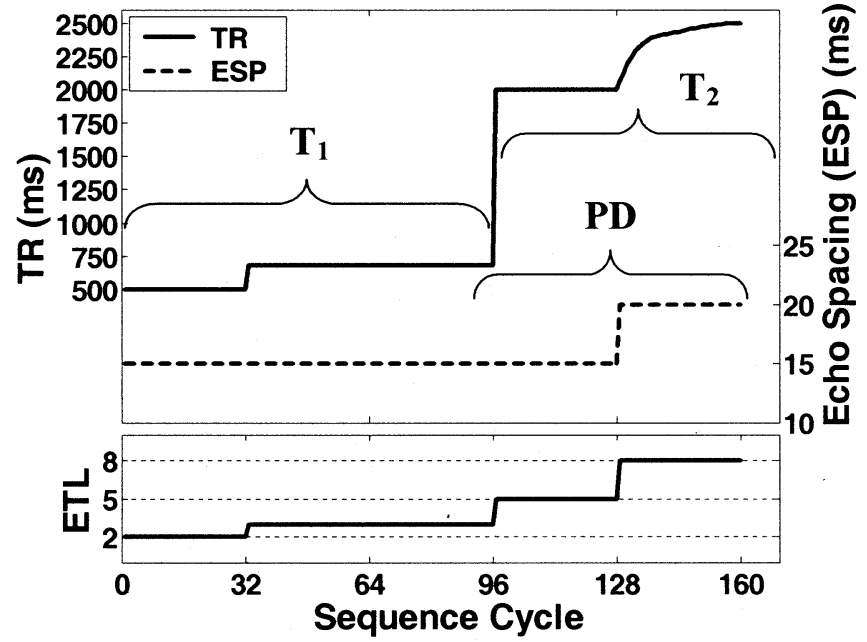

(a)

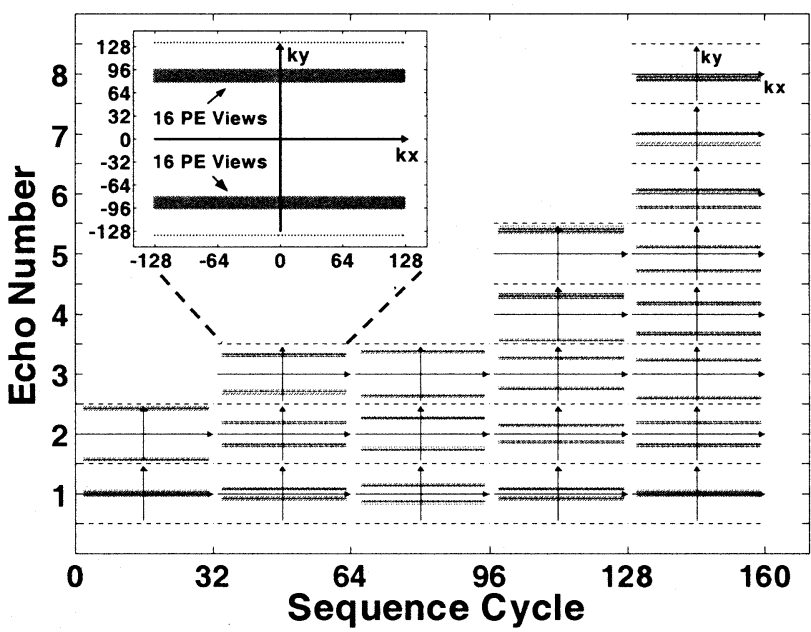

(b)

Fig. 10. Optimized parameter variation and PE schemes for a 3-contrast FSE combo acquisition ( $T_{1^{-}}$, PD-, $\left.T_{2}\right)$ with 160 sequence cycles (a) Curves of variation for TR (top, solid), ESP (top, dashed), and ETL (bottom, solid). (b) PE scheme showing the PE views in $k$-space that are generated with each echo for sets of 32 sequence cycles. Inset in the upper left corner of (b) shows an enlarged $k$-space diagram for one echo. In the top part of (a), it is illustrated which parts of the generated data were utilized to reconstruct a $T_{1^{-}}, \mathrm{PD}-$, and $T_{2}$-weighted image, respectively.

in Fig. 13. Images in Fig. 13(a)-(f) correspond to slice locations $z=-30,-20,-10,+10,+30$, and +60 , respectively. Image quality and level of artifacts are very similar to the ones in the image shown in Fig. 11(a) obtained with the same variation scheme and corresponding to slice location $z=0$. Similar observations were made for the corresponding PD- and $T_{2}$-weighted images. This shows that parameter variation and PE schemes optimized for a specific set of phantom data yield similar results, when applied to phantom data with the same tissue types, but different (brain) structures. It should be noted though that the level of artifacts for these results depends on the spatial frequency content of the selected set of phantom data.

\section{E. Quantitative Results From Optimization and Evaluation}

In this section, the three quantitative optimization criteria are mathematically defined, and quantitative results for these criteria and for the evaluation of combo acquisitions are provided.

1) Quantitative Optimization Criteria:

a) Energy of Residue PSF: Varying acquisition parameters TR, TE, and/or ETL clearly effects the PSF. The PSF in MRI can be considered as the inverse Fourier transform of a filter $H(\vec{k})$ that multiplies the $k$-space data. In the image domain, this is equivalent to convolution of the transformed data with a filter $h(\vec{r})$, where $h$ is the PSF. To analyze the PSF in standard and combo acquisitions, respectively, simulations with phantom data of single object points with voxel fractions equal to 1 (discrete delta functions) were run for each tissue. Since the PSF is an important characteristic for MR imaging techniques, the energy of the "residue PSF" $E$ ( PSF $\left._{\text {res }}\right)$ was used as one of the quantitative criteria to be minimized for an optimized combo acquisition. In this context, the energy of the residue PSF, i.e., the energy of the difference in PSF between combo and corresponding standard acquisitions, was defined as (assuming that the PE direction is along the $y$ axis)

$$
\begin{aligned}
& E\left(\mathrm{PSF}_{\mathrm{res}}\right)=\sum_{i=1}^{N_{\text {contrast }}} \sum_{j=1}^{N_{\text {tissues }}} w_{i j}^{\mathrm{PSF}} \\
& \cdot \int_{-\mathrm{FOV}_{\mathrm{y}} / 2}^{+\mathrm{FOV}_{y} / 2}\left|\operatorname{PSF}(y)_{i j}^{\text {combo }}-\operatorname{PSF}(y)_{i j}^{\text {standard }}\right|^{2} d y
\end{aligned}
$$

where $N_{\text {contrast }}$ is the number of different contrasts in a multicontrast acquisition, $N_{\text {tissues }}$ is the number of tissues used in 


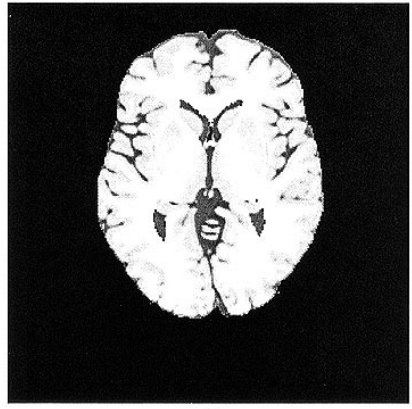

(a)

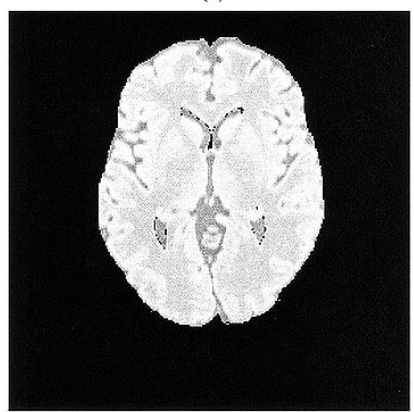

(c)

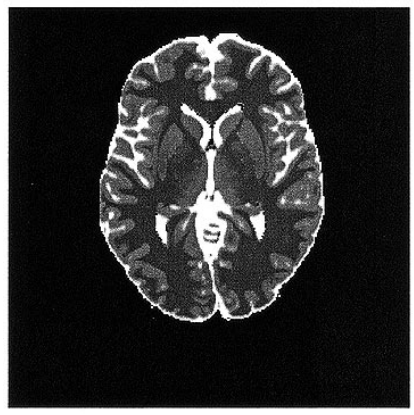

(e)

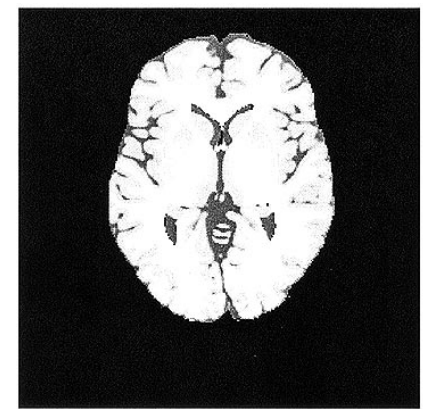

(b)

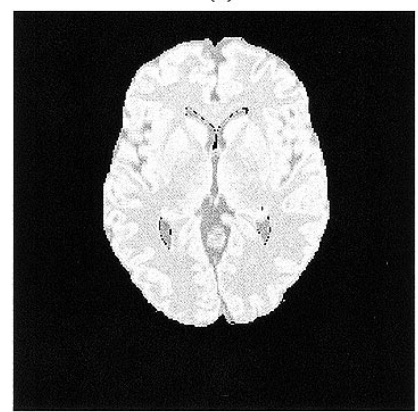

(d)

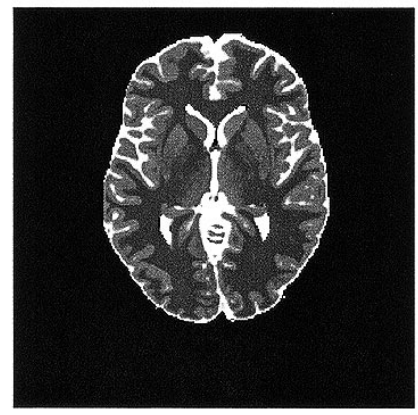

(f)
Fig. 11. Simulated images for three different contrasts, $T_{1^{-}}$, PD-, and $T_{2}$-weighted: Images in (a), (c), and (e) from a 3-contrast FSE combo acquisition using the variation scheme in Fig. 10, and images in (b), (d), and (f) from corresponding standard acquisitions (a $T_{1}$-weighted $\mathrm{SE}$ and a dual-contrast FSE sequence) with parameters from Table II. The acquisition parameters TR, ESP, and ETL were varied during the generation of $k$-space data. Predicted scan time was reduced by $30 \%$ with the combo acquisition.

phantom data, and $w_{i j}^{\mathrm{PSF}}$ are the weighting factors that depend on the respective diagnostic importance of a contrast, and on the signal level from the corresponding standard acquisition of a tissue for each contrast. These weighting factors were chosen to account for the "visibility" of artifacts for different tissues in images of a particular contrast. For example, artifacts in tissues with larger signal levels for a specific contrast are usually more visible and thus have to be weighted more strongly. Thus, for contrast $i$ and tissue $j$, the weight $w_{i j}^{\mathrm{PSF}}$ was computed from its corresponding magnitude of signal level ( $k$-space data weighting) for the zero PE view denoted by $\left.\operatorname{signal}\left(k_{y}\right)_{i j}\right|_{k_{y}=0}$. The larger the signal level, the larger the assigned weight $w_{i j}^{\mathrm{PSF}}$. More specifically, normalized weighting factors $w_{i j}^{\mathrm{PSF}}$ for $E\left(\mathrm{PSF}_{\mathrm{res}}\right)$ were chosen as

$$
w_{i j}^{\mathrm{PSF}}=\frac{\left.\operatorname{signal}\left(k_{y}\right)_{i j}\right|_{k_{y}=0}}{\max _{j}\left(\left.\operatorname{signal}\left(k_{y}\right)_{i j}\right|_{k_{y}=0}\right)} .
$$

b) Energy of Residue Profiles: Changes of the PSF do not always directly translate into changes of the visual appearance of objects in an image (e.g., for homogeneous tissue areas). Thus, another quantitative criterion for the optimization process was derived from image profiles across sharp tissue boundaries (edges). Such profiles were generated from images of a phantom that consisted of two adjacent rows of three consecutive homogeneous tissue blocks of size $60 \times 60$ points along in-plane coordinates. The distribution of tissues WM, GM, and CSF over the six blocks was chosen to obtain profiles along the $\mathrm{PE}$ direction in reconstructed images for all possible tissue combinations. To yield a quantitative criterion, the energy of "residue profiles" across sharp tissue boundaries $E$ (profiles $_{\text {res }}$ ) was computed. Similar to the residue PSF, it was expressed as

$$
\begin{aligned}
& E\left(\operatorname{profiles}_{\mathrm{res}}\right)=\sum_{i=1}^{N_{\text {contrast }}} \sum_{j=1}^{N_{\text {tissueComb }}} w_{i j}^{\text {profiles }} \\
& \cdot \int_{-\mathrm{FOV}_{\mathrm{y}} / 2}^{+\mathrm{FOV}_{y} / 2}\left|\operatorname{profile}(y)_{i j}^{\text {combo }}-\operatorname{profile}(y)_{i j}^{\text {standard }}\right|^{2} d y
\end{aligned}
$$

where, $N_{\text {tissueComb }}$ refers to the number of tissue combinations, for which profiles were obtained. All other notations are as defined for $E\left(\mathrm{PSF}_{\mathrm{res}}\right)$ in (5). In (7), normalized weighting factors $w_{i j}^{\text {profiles }}$ for contrast $i$ and tissue combination $j$ (with tissues $j_{1}$ and $j_{2}$ ) were selected as

$$
\begin{aligned}
& w_{i j}^{\text {profiles }}=\frac{\alpha}{\max _{j}(\alpha)} \\
& \alpha=\frac{1}{\left|\operatorname{signal}\left(k_{y}\right)_{i \text { tissue } j_{1}}\right|_{k_{y}=0}-\left.\operatorname{signal}\left(k_{y}\right)_{i \text { tissue } j_{2} \mid}\right|_{k_{y}=0} \mid} .
\end{aligned}
$$

As expressed in (8), the energy of a residue profile for a specific tissue combination was weighted according to the inverse of the absolute value of the difference in signal level at $k_{y}=0$ between the two tissues involved. The smaller this difference, the larger the assigned weight $w_{i j}^{\text {profiles }}$. This was chosen to weight residue profiles from tissues with similar signal levels, which are converted into similar image intensities after IFFT, more strongly, since deviations from similar intensities might yield less discernible image objects.

c) Energy of Residue Images: Finally, as a third quantitative criterion for assessing the optimization of combo acquisitions, the energy of "residue images" $E$ (images res) was introduced. $E$ (images (imes $_{\text {) }}$ is the weighted sum of the energies of the difference images obtained by subtracting the images from combo and corresponding standard acquisitions from each other. It was formulated as

$$
\begin{aligned}
& E\left(\text { images }_{\mathrm{res}}\right)=\sum_{i=1}^{\mathrm{N}_{\text {contrast }}} w_{i}^{\text {images }}
\end{aligned}
$$

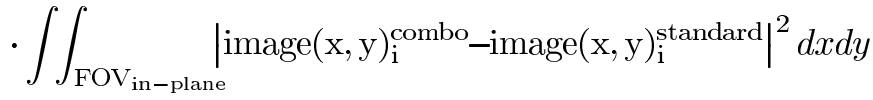

where, the summation of energies was carried out over all images of different contrasts weighted by factors $w_{i}^{\text {images }}$. 


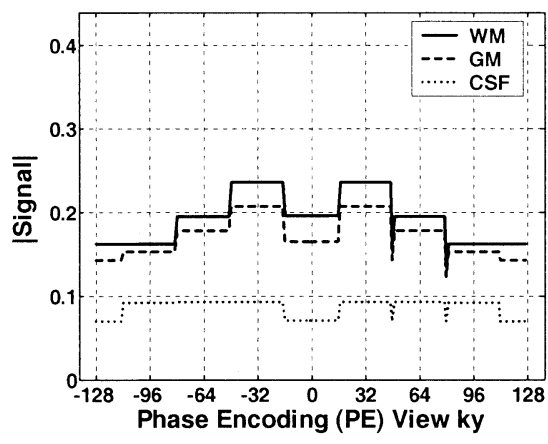

(a)

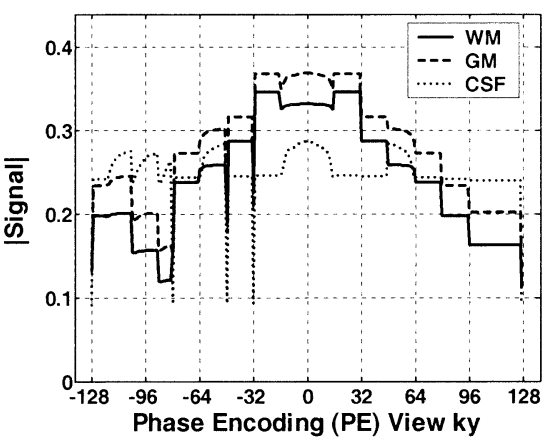

(b)

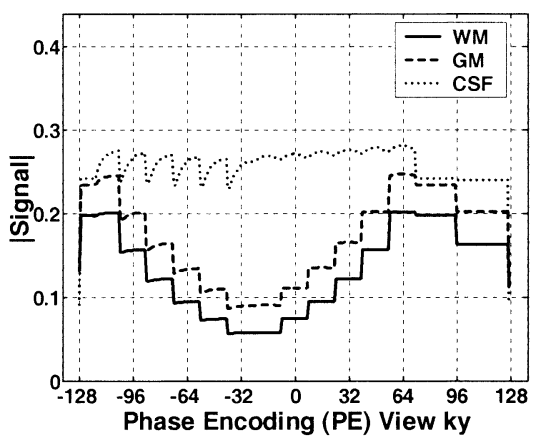

(c)

Fig. 12. Signal levels for tissues WM, GM, and CSF for (a) a $T_{1^{-}}$, (b) a PD-, and (c) a $T_{2}$-weighted image of the 3-contrast FSE combo acquisition in Fig. 11.

TABLE III

SCAN TIME REDUCTIONS FOR SE AND FSE COMBo ACQUisITIONS $($ NEX $=1$ )

\begin{tabular}{l|c|c|c}
\hline Contrasts & 2-Contrast SE Combo & 3-Contrast SE Combo & 3-Contrast FSE Combo \\
\hline \hline $\mathrm{T}_{1}-\mathrm{T}_{2}$ & $47 \%, 52 \%$ & - & - \\
\hline $\mathrm{T}_{1}-\mathrm{PD}$ & $47 \%, 52 \%$ & - & - \\
\hline $\mathrm{PD}-\mathrm{T}_{2}$ & $25 \%$ & - & - \\
\hline $\mathrm{T}_{1}-\mathrm{PD}-\mathrm{T}_{2}$ & - & $31 \%$ & $30 \%$ \\
\hline
\end{tabular}

In general, all contrasts were weighted equally, so that for $E$ (images (ims $) w_{i}^{\text {images }}=1, i=1,2, \ldots, N_{\text {contrast }}$.

Values for the three quantitative criteria energy of residue PSF $E$ ( PSF $_{\text {res }}$ ), energy of residue profiles across sharp tissue

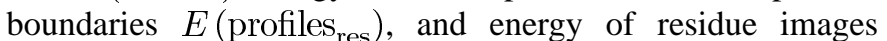
$E$ (images (res ) used to assess the optimization of combo acquisitions, are shown in Table IV for the optimized combo acquisitions presented so far. The corresponding weighting factors used to compute these quantities are presented in Table V.

To illustrate how minimization of the three criteria leads to optimization of a combo acquisition protocol, values for $E\left(\mathrm{PSF}_{\text {res }}\right), E$ (profiles $_{\text {res }}$ ), and $E$ (images (ies ) for optimized 3 -contrast FSE combo acquisition protocols and for (nonoptimized) combo scans with different parameter variation and/or PE schemes are plotted in Fig. 14. For display purposes, residue quantities were scaled to lie within the same range of values. As seen from Fig. 14, optimized combo acquisitions minimized the three selected quantities compared with nonoptimized combo scans (see the plateaus of small values in all three curves of Fig. 14). Certainly, the amount of predicted scan time reduction does play a role in selecting a specific protocol as "the optimized combo acquisition," since residue quantities could be further minimized by decreasing the time savings of an acquisition. In this paper, predicted scan time reductions for a FSE combo acquisition of at least, $30 \%$ were targeted. Thus, acquisition protocol 17 in Fig. 14 was selected and was shown in Fig. 10.

2) Contrast Measurements: To evaluate the preservation of contrast with combo acquisitions, image contrast was measured by computing ratios of average image intensities in selected regions of interest (ROIs) of homogeneous tissue content. The size of each ROI was $5 \times 5$ pixels. Larger contiguous ROIs could not be found for each tissue. Ratios of average image inten- sities were calculated for all tissue combinations (WM-GM, WM-CSF, and GM-CSF). Contrast measurements for images from standard and combo acquisitions are presented in Table VI. Each table entry contains three ratios of image intensities for tissue combinations WM-GM, WM-CSF, and GM-CSF. Since the results of these measurements for images from standard and combo acquisitions were nearly identical, no contrast degradation was observed.

3) Analysis of PSF and Test of Robustness: In addition to its use as a quantitative optimization criterion, the PSF was analyzed by computing the respective full-width at half maximum (FWHM) and full-width at tenth maximum (FWTM) of its magnitude. Results from these computations were compared for standard and combo acquisitions to detect any changes of the PSF. As a sample case, the real part of the PSF along the phase encoding direction for the tissues WM, GM, and CSF in a $T_{1}$-weighted image from the 3 -contrast $\mathrm{SE}$ combo acquisition with the variation scheme in Fig. 7 and from the corresponding standard acquisition is shown in Fig. 15. For both cases the PSF looks very similar, though for the combo acquisition the (real part of the) PSF has side lobes of small amplitudes as can be seen in Fig. 15(a), which are absent in the perfect PSF of Fig. 15(b). Results for FWHM and FWTM of the magnitude of the PSF in pixels for images from standard acquisitions and combo acquisitions are summarized in Table VII (FWHM) and Table VIII (FWTM). Each table entry contains three values for tissues WM, GM, and CSF (in this order) computed at subpixel resolution obtained through interpolation. Results in Table VII and in Table VIII indicate a slightly widened center lobe of the PSF for images from combo compared with SE standard acquisitions. As a consequence, some blurring of small image objects occurred. Furthermore, since side lobes of the PSF were induced, ringing artifacts across sharp tissue boundaries were observed. These 


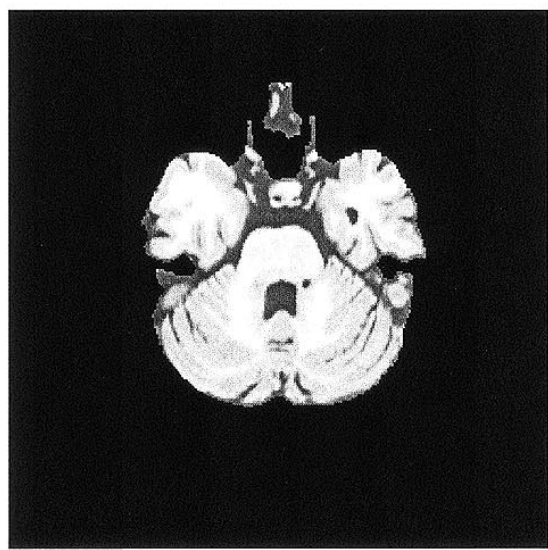

(a)

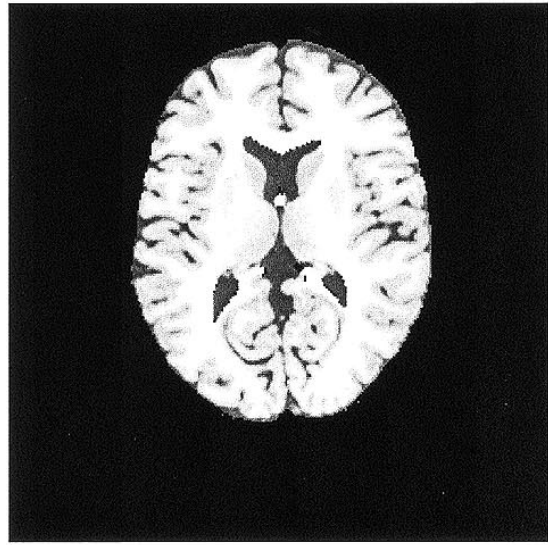

(d)

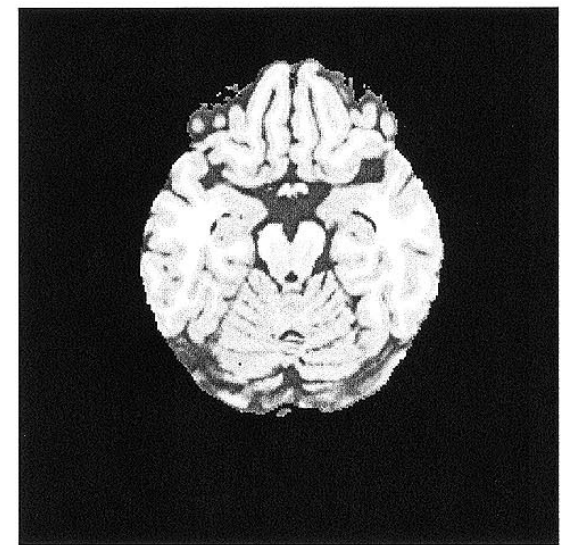

(b)

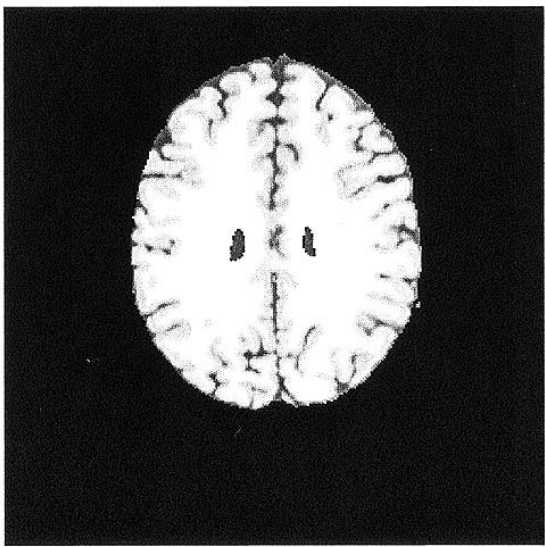

(e)

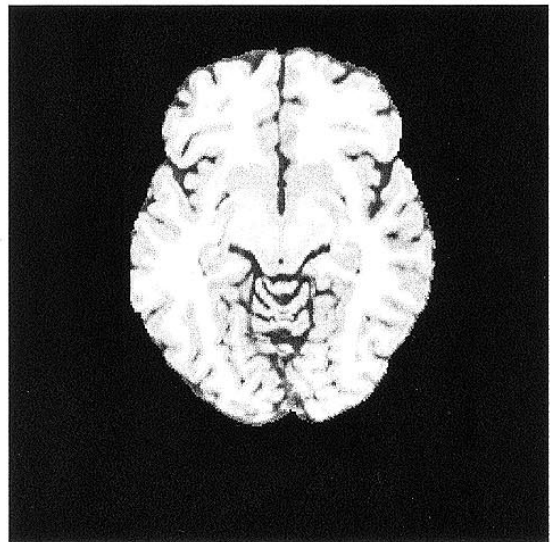

(c)

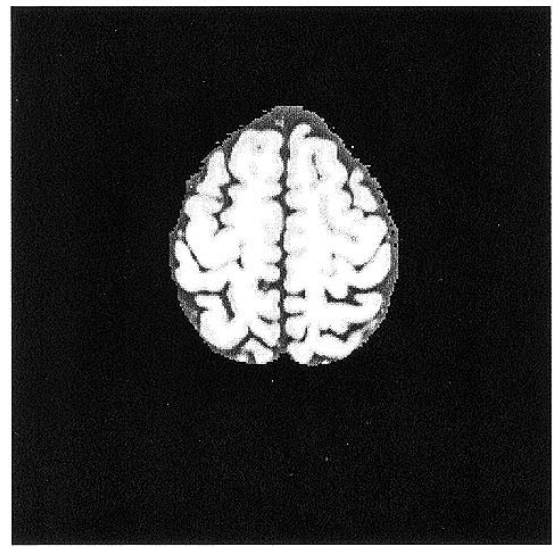

(f)

Fig. 13. Simulated $T_{1}$-weighted images of six axial slices from the 3-contrast FSE combo acquisition using the variation scheme in Fig. 10. Images in (a) - (f) correspond to slice locations $z=-30,-20,-10,+10,+30$, and +60 , respectively. Image quality and level of artifacts are very similar to the ones in the image shown in Fig. 11(a) obtained with the same variation scheme and corresponding to slice location $z=0$.

TABLE IV

QuANTITATIVE OPtIMIZATION CRITERIA FOR SE AND FSE COMBo ACQUISITIONS: ENERgy OF ReSidue PSF E (PSF res), ENERGY OF RESIDUE PROFILES ACROSS SHARP TISSUE BOUNDARIES $E$ ( profiles $_{\text {res }}$ ), AND ENERGY OF RESIDUE IMAGES $E$ (images $_{\text {res }}$ )

\begin{tabular}{l|c|c|c}
\hline Criterion & 2-Contrast SE Combo & 3-Contrast SE Combo & 3-Contrast FSE Combo \\
\hline \hline$E\left(\right.$ PSF $\left._{\text {res }}\right)$ & 0.0312 & 0.0512 & 0.0073 \\
\hline$E\left(\right.$ profiles $\left._{\text {res }}\right)$ & 0.0244 & 0.0690 & 0.0330 \\
\hline${\left.\text { E } \text { (images }_{\text {res }}\right)}$ & 18.029 & 10.400 & 7.607 \\
\hline
\end{tabular}

effects are due to nonuniform $k$-space data weighting and were expected. In contrast, results for FSE combo and reference scans were rather similar (see Tables VII and VIII). Thus, corresponding artifacts were similar as well.

In general, optimization of combo scans for selected contrasts was tissue dependent, therefore mainly dependent on relaxation times $T_{1}$ and $T_{2}$. Hence, the robustness of an optimized combo acquisition protocol was tested by examining the PSF for a wide range of relaxation times, when using the same protocol. For this test, the FWHM and the FWTM of the magnitude of the PSF were determined for all combinations of $T_{1} \in[250,5000]$ and $T_{2} \in[40,2500]$, but with a fixed value for the spin density $\left(\rho=0.69=\rho_{\mathrm{GM}}\right)$. Samples for $T_{1}$ and $T_{2}$ were chosen to include typical values for other tissues most commonly encoun- tered in MRI, such as fat, muscle, and blood, and some extreme values. Selected results from these experiments for the 3-contrast FSE combo acquisition with the variation schemes shown in Fig. 10 are presented in Table IX (FWHM) and in Table X (FWTM). In addition, the minimum, maximum, mean, and standard deviation $\sigma$ of the FWHM and the FWTM with respect to all different tissues were computed for each contrast. These results are summarized in Table XI. In general, the FWHM and FWTM of the (magnitude of the) PSF of tissues with large transverse relaxation times $\left(T_{2} \geq 200\right)$ were more robust to variations of acquisition parameters compared with tissues with smaller values for $T_{2}$ (means for data with $T_{2} \geq 200$ were reduced by $6 \%-35 \%$ and standard deviations were reduced by $80 \%-90 \%$ ). Variations with respect to $T_{1}$ for a given $T_{2}$ only led 
TABLE V

Weighting Factors $W$ Used to COMPUTE THE THREe QuANTITATIVE OPTIMIZATION CRITERIA FOR COMBo ACQUisitions FOR EACH CONTRAST AND TISSUES WM, GM, AND CSF

\begin{tabular}{|c|c|c|c|c|}
\hline Criterion & Contrast & 2-Contrast SE Combo & 3-Contrast SE Combo & 3-Contrast FSE Combo \\
\hline \multirow{3}{*}{$E\left(P S F_{r e s}\right)$} & $\mathbf{T}_{1}$ & $1.0,0.86,0.38$ & $1.0,0.86,0.38$ & $1.0,0.85,0.36$ \\
\hline & PD & - & $0.89,1.0,0.80$ & $0.91,1.0,0.78$ \\
\hline & $\mathbf{T}_{2}$ & $0.24,0.37,1.0$ & $0.24,0.37,1.0$ & $0.27,0.41,1.0$ \\
\hline \multirow{3}{*}{$E\left(\right.$ profiles $\left._{\text {res }}\right)$} & $T_{1}$ & $1.0,0.23,0.30$ & $1.0,0.23,0.30$ & $1.0,0.24,0.32$ \\
\hline & PD & - & $0.85,1.0,0.46$ & $1.0,0.81,0.45$ \\
\hline & $\mathbf{T}_{2}$ & $1.0,0.17,0.21$ & $1.0,0.17,0.21$ & $1.0,0.19,0.23$ \\
\hline \multirow{3}{*}{$E\left(\right.$ images $\left._{\text {res }}\right)$} & $T_{1}$ & 1 & 1 & 1 \\
\hline & PD & - & 1 & 1 \\
\hline & $\mathbf{T}_{2}$ & 1 & 1 & 1 \\
\hline
\end{tabular}

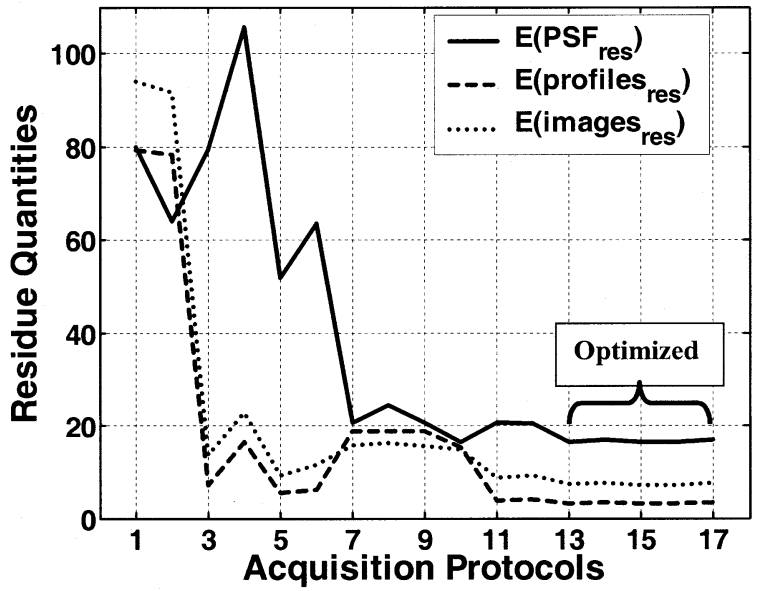

Fig. 14. Plot of quantitative optimization criteria $E\left(\mathrm{PSF}_{\text {res }}\right)$, $E$ (profiles res ), and $E$ (images $_{\text {res }}$ ) for optimized and nonoptimized 3-contrast FSE combo acquisitions. Residue quantities were scaled to lie within the same range of values for display purposes. Note that optimized combo scans minimize all three quantities (plateau part of the curves) compared with nonoptimized protocols.

to minor changes of the FWHM and the FWTM of the (magnitude of the) PSF. For a given $T_{2}$, the maximum change of the FWHM with $T_{1}$ was less than 5\%, and the maximum change for the FWTM was $14 \%$, though for most values of $T_{2}$ less than $10 \%$. Note that the mean values of the FWHM and the FWTM for each contrast lie in the range of these quantities obtained for tissues WM, GM, and CSF (see Table XI, Table VI, and Table VII). Thus, for most tissues encountered in MRI the widening of the center lobe of the PSF induced by combo acquisitions should be similar to the widening observed for the tissues selected in this paper. If one considers the widening of the center lobe of the PSF as one suitable measure for the resulting level of artifacts, applying an optimized (3-contrast FSE) combo acquisition to imaging different tissues should yield images with levels of artifacts similar to those of the images presented in this paper. Only for tissues with relative short transverse relaxation times $\left(T_{2} \leq 60\right)$, such as muscle or fat, the level of artifacts may be increased. Hence, optimized combo acquisitions exhibit certain robustness with respect to different relaxation times, although the optimization process of the combo acquisition was carried out for a specific set of tissues.
4) Quantification of the SNR: As a final evaluation step the SNR was quantified by adding complex Gaussian white noise to simulated $k$-space data and computing the SNR of the resulting magnitude images. In general, the MR signal for 2-D imaging with the addition of noise can be written as [40], [41]

$$
y\left(k_{x}, k_{y}\right)=s\left(k_{x}, k_{y}\right)+n\left(k_{x}, k_{y}\right)
$$

where, $s\left(k_{x}, k_{y}\right)$ is the uncontaminated (complex) signal and $n\left(k_{x}, k_{y}\right)$ is a complex Gaussian white noise. After applying the inverse Fourier transform to $y\left(k_{x}, k_{y}\right)$, the noise is, again complex Gaussian white noise, since the Fourier transform is an unitary transformation. However, for the magnitude image, this type of noise addition results in a Rician noise distribution [40], [42]. For the SNR measurements, an estimate of the variance, $\sigma^{2}$, was obtained from the average (magnitude) image intensity in a ROI of size $32 \times 32$ pixels outside of the brain area corrected by a factor accounting for the Rician distribution of the noise [37]. Signal was computed as average image intensity of a ROI of size $12 \times 12$ pixels of homogeneous white matter. The measurement was repeated 50 times (each time a newly generated noise matrix was added to the uncontaminated signal), and values were averaged for all these cases. Results for SNR measurements for noise with variance $\sigma=1.5$ and acquisition with NEX $=1$ are given in Table XII for images from standard and combo acquisitions. As seen from Table XII, SNR values were the same for images from combo and standard scans.

\section{F. Images With Pathology}

The performance of combo acquisitions in the case of patients with pathology is of great importance, if combined scans are to be effectively translated to day-to-day imaging. To account for cases of brain pathology, simulations were performed for brain slice data with lesions of MS. The corresponding dataset was also obtained from the Brain Imaging Centre in Montreal [36]. Physical parameters for MS lesions were chosen as $T_{1}=1428 \mathrm{~ms}, T_{2}=139 \mathrm{~ms}$ [43], and $\rho=0.8 * 10^{31} \mathrm{~cm}^{-3}$. Images of an axial slice with known distribution of MS lesions from a 3-contrast FSE combo acquisition are presented in Fig. 16 together with images from corresponding standard acquisitions. Arrows indicate sample locations of MS lesions in Fig. 16(e) and (f), respectively. As can be seen in Fig. 16, there is no visual difference in the appearance of MS lesions 
TABLE VI

Contrast Ratios of Tissues WM, GM, AND CSF IN IMAGES From SE AND FSE COMBo ACQUisitions AND CORRESPONDING STANDARD ACQUISITIONS

\begin{tabular}{l|c|c|c|c|c}
\hline $\begin{array}{c}\text { Contrast of } \\
\text { Image }\end{array}$ & $\begin{array}{c}\text { Standard } \\
\text { For SE Combo }\end{array}$ & $\begin{array}{c}\text { 2-Contrast SE } \\
\text { Combo }\end{array}$ & $\begin{array}{c}\text { 3-Contrast SE } \\
\text { Combo }\end{array}$ & $\begin{array}{c}\text { Standard } \\
\text { For FSE Combo }\end{array}$ & $\begin{array}{c}\text { 3-Contrast FSE } \\
\text { Combo }\end{array}$ \\
\hline $\mathrm{T}_{1}$ & $1.17: 2.61: 2.24$ & $1.17: 2.61: 2.23$ & $1.17: 2.61: 2.23$ & $1.18: 2.76: 2.34$ & $1.18: 2.77: 2.33$ \\
\hline $\mathrm{PD}$ & $0.89: 1.11: 1.25$ & - & $0.89: 1.12: 1.25$ & $0.90: 1.15: 1.29$ & $0.89: 1.15: 1.29$ \\
\hline $\mathrm{T}_{2}$ & $0.65: 0.24: 0.37$ & $0.65: 0.24: 0.38$ & $0.65: 0.24: 0.37$ & $0.67: 0.27: 0.41$ & $0.68: 0.27: 0.40$ \\
\hline
\end{tabular}
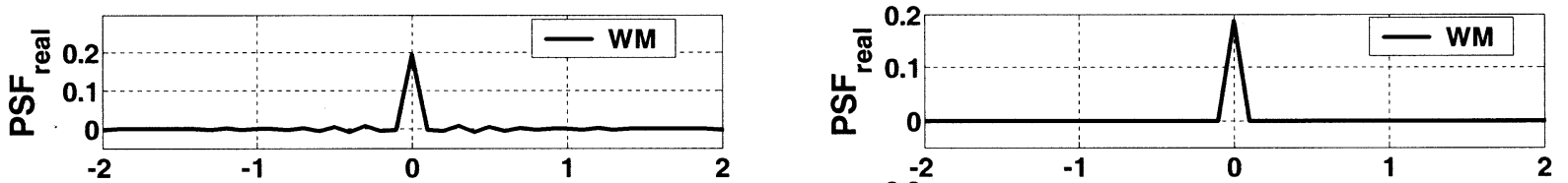

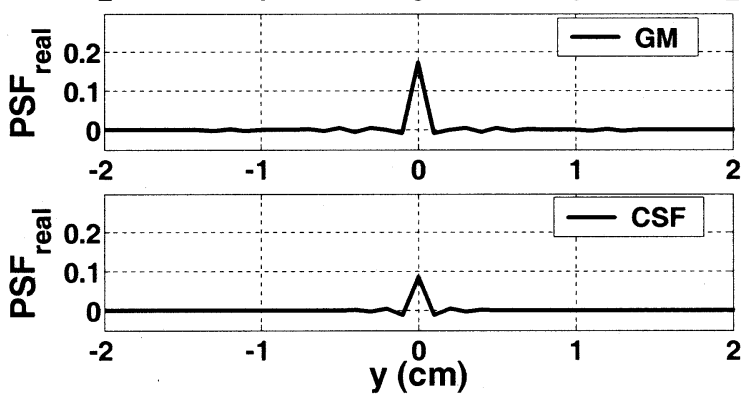

(a)

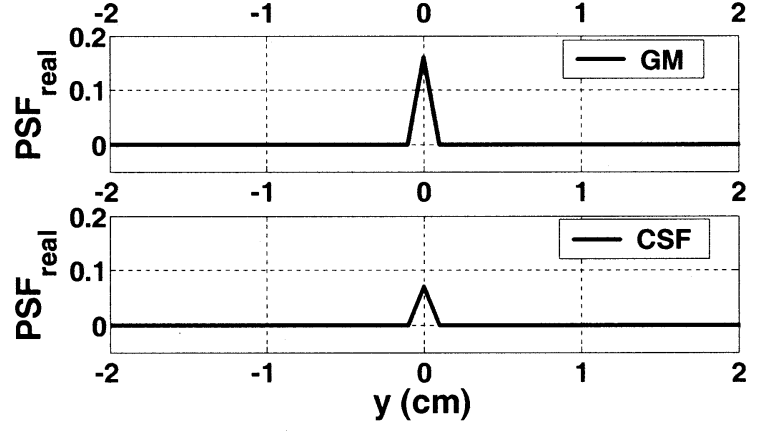

(b)

Fig. 15. Real part of PSF along phase encoding direction for tissues WM, GM, and CSF (top to bottom) in $T_{1}$-weighted images for (a) a 3-contrast SE combo acquisition [Fig. 8(a)] and (b) corresponding standard acquisition [Fig. 8(b)].

TABLE VII

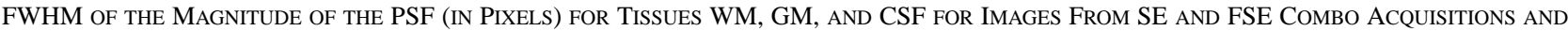
CORRESPONDING STANDARD ACQUISITIONS

\begin{tabular}{l|c|c|c|c|c}
\hline $\begin{array}{l}\text { FWHM } \\
\text { PSF }\end{array}$ & $\begin{array}{c}\text { Standard } \\
\text { For SE Combo }\end{array}$ & $\begin{array}{c}\text { 2-Contrast SE } \\
\text { Combo }\end{array}$ & $\begin{array}{c}\text { 3-Contrast SE } \\
\text { Combo }\end{array}$ & $\begin{array}{c}\text { Standard } \\
\text { For FSE Combo }\end{array}$ & $\begin{array}{c}\text { 3-Contrast FSE } \\
\text { Combo }\end{array}$ \\
\hline $\mathrm{T}_{1}$ & $1.00,1.00,1.00$ & $1.32,1.23,1.02$ & $1.01,1.05,1.13$ & $1.00,1.00,1.00$ & $1.09,1.06,1.00$ \\
\hline $\mathrm{PD}$ & $1.00,1.00,1.00$ & - & $1.18,1.24,1.35$ & $1.23,1.18,1.01$ & $1.22,1.17,1.01$ \\
\hline $\mathrm{T}_{2}$ & $1.00,1.00,1.00$ & $1.04,1.04,1.45$ & $1.32,1.21,1.04$ & $1.23,1.18,1.01$ & $1.32,1.23,1.02$ \\
\hline
\end{tabular}

TABLE VIII

FWTM of the MAgnitude of THE PSF (IN PiXels) For Tissues WM, GM, AND CSF FOR IMAGES From SE AND FSE COMBo ACQUiSITIONS AND CORRESPONDING STANDARD ACQUISITIONS

\begin{tabular}{l|c|c|c|c|c}
\hline $\begin{array}{l}\text { FWHM } \\
\text { PSF }\end{array}$ & $\begin{array}{c}\text { Standard } \\
\text { For SE Combo }\end{array}$ & $\begin{array}{c}\text { 2-Contrast SE } \\
\text { Combo }\end{array}$ & $\begin{array}{c}\text { 3-Contrast SE } \\
\text { Combo }\end{array}$ & $\begin{array}{c}\text { Standard } \\
\text { For FSE Combo }\end{array}$ & $\begin{array}{c}\text { 3-Contrast FSE } \\
\text { Combo }\end{array}$ \\
\hline $\mathrm{T}_{1}$ & $1.61,1.61,1.61$ & $2.87,2.65,1.68$ & $1.65,1.74,2.81$ & $1.61,1.61,1.61$ & $1.89,1.80,1.61$ \\
\hline $\mathrm{PD}$ & $1.61,1.61,1.61$ & - & $2.45,2.85,4.71$ & $2.71,2.47,1.63$ & $2.69,2.48,1.65$ \\
\hline $\mathrm{T}_{2}$ & $1.61,1.61,1.61$ & $1.74,1.72,4.69$ & $3.05,2.57,1.74$ & $2.71,2.47,1.63$ & $3.18,3.04,1.67$ \\
\hline
\end{tabular}

in the images in Fig. 16(a), (c), and (e) acquired with variable acquisition parameters from corresponding images in Fig. 16(b), (d), and (f) obtained with standard scans. Since in combo acquisitions the lowest spatial frequencies of each image of different contrast are acquired with the same settings as in corresponding standard acquisitions, overall contrast of lesions with respect to surrounding brain tissues is preserved.

\section{DISCUSSION}

In the following subsections, we discuss important implications and challenges for the practical use of combo acquisitions.

\section{A. Steady-State Considerations}

In standard SE and FSE sequences, the transverse magnetization reaches steady state during all sequence cycles. Changing acquisition parameters for the acquisition of each PE view may not let the spin system reach steady state, especially since variable TR entails varying longitudinal relaxation toward the unperturbed equilibrium magnetization $M_{0}$. However, signal oscillations during simulated data acquisition with combo acquisitions were found to be numerically small, when TR was varied continuously. When the variation of TR contained discontinuities [e.g., see Fig. 10(a)], only the signal of the first 
TABLE IX

FWHM OF THE MAGNitude OF THE PSF (IN PIXelS) FOR SELECTED $T_{1} \in[250,5000]$ AND $T_{2} \in[40,2500]$ FOR THE 3-CONTRAST FSE COMBO ACQUISITION SHOWN IN FIG. 10

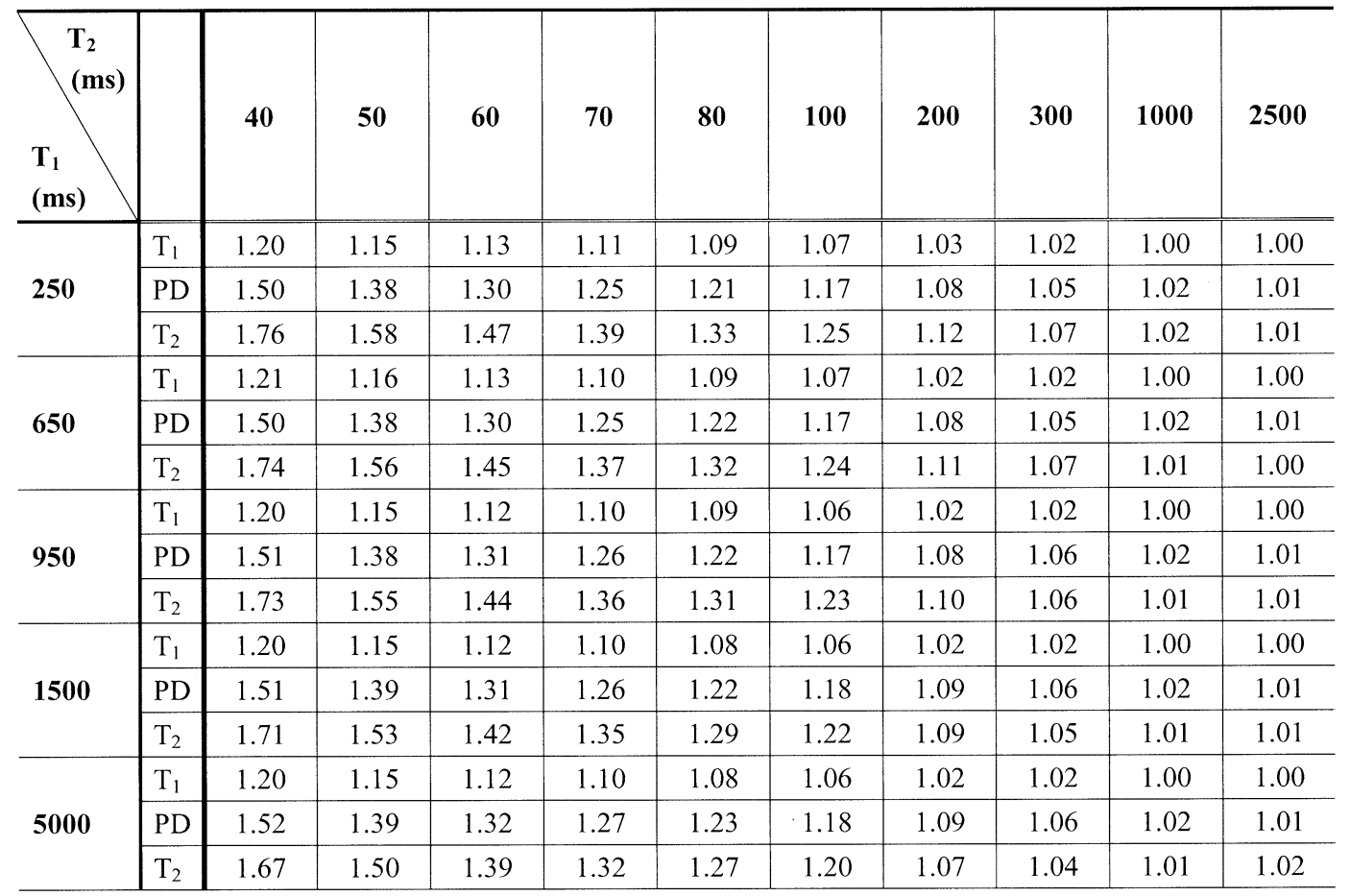

TABLE X

FWTM OF THE MAGNITUde Of THE PSF (IN PIXELS) FOR SELECTED $T_{1} \in[250,5000]$ AND $T_{2} \in[40,2500]$ FOR THE 3-CONTRAST FSE COMBO ACQUISITION SHOWN IN FIG. 10

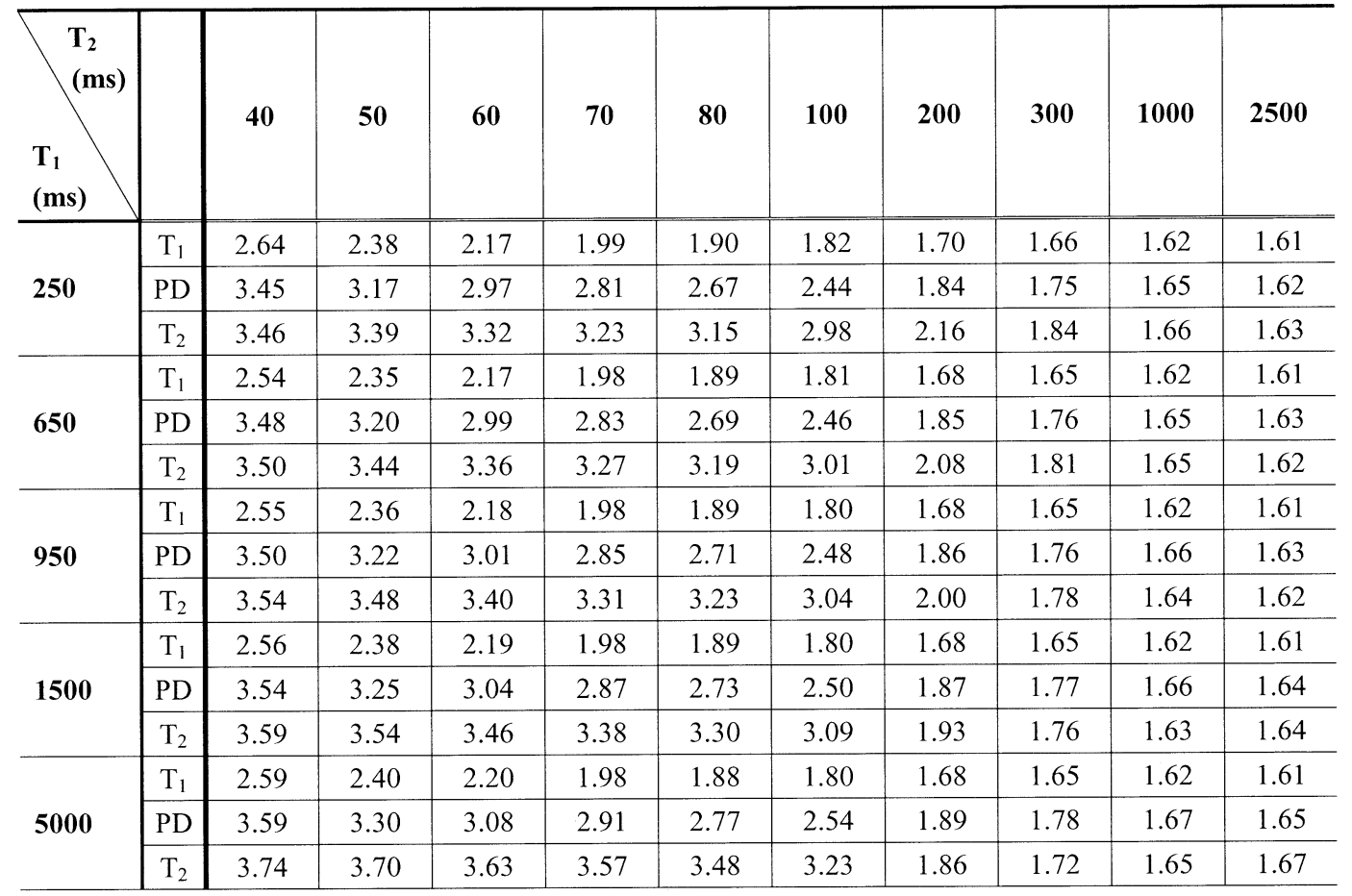

sequence cycle immediately following such a jump in TR was affected [see Fig. 12]. Thus, maintaining steady state did not pose a problem in spin echo based combo acquisitions with the ranges and variation curves selected in this study.

\section{B. Motion Artifacts}

To evaluate the clinical utility of combo acquisitions, motion artifacts specific to combo scans have to be considered. For gen- 
TABLE XI

Minimum, Maximum, Mean, AND Standard DeViation, $\sigma$, OF THE FWHM AND the FWTM (IN PiXels) FOR $T_{1} \in[250,450,650,850,950,1200,1500,2500,4000,5000]$ AND $T_{2} \in[40,50,60,70,80,100,200,300,1000,2500]$ FOR THE 3-CONTRAST FSE COMBO ACQUISITION SHOWN IN FIG. 10

\begin{tabular}{l|c|c|c|c|c|c|c|c}
\hline $\begin{array}{l}\text { Contrast } \\
\text { of Image }\end{array}$ & $\begin{array}{c}\text { Minimum } \\
\text { FWHM }\end{array}$ & $\begin{array}{c}\text { Maximum } \\
\text { FWHM }\end{array}$ & $\begin{array}{c}\text { Mean } \\
\text { FWHM }\end{array}$ & $\begin{array}{c}\sigma \text { of } \\
\text { FWHM }\end{array}$ & $\begin{array}{c}\text { Minimum } \\
\text { FWTM }\end{array}$ & $\begin{array}{c}\text { Maximum } \\
\text { FWTM }\end{array}$ & $\begin{array}{c}\text { Mean } \\
\text { FWTM }\end{array}$ & $\begin{array}{c}\sigma \text { of } \\
\text { FWTM }\end{array}$ \\
\hline \hline $\mathrm{T}_{1}$ & 1.00 & 1.21 & 1.08 & 0.06 & 1.61 & 2.64 & 1.94 & 0.32 \\
\hline $\mathrm{PD}$ & 1.01 & 1.52 & 1.20 & 0.16 & 1.62 & 3.59 & 2.48 & 0.66 \\
\hline $\mathrm{T}_{2}$ & 1.00 & 1.76 & 1.27 & 0.23 & 1.62 & 3.74 & 2.73 & 0.81 \\
\hline
\end{tabular}

TABLE XII

SNR MEASUREMENTS IN IMAges From SE AND FSE COMBO ACQUiSITIONS AND CORRESPONDING STANDARD ACQUISITIONS

\begin{tabular}{l|c|c|c|c|c}
\hline $\begin{array}{c}\text { Contrast of } \\
\text { Image }\end{array}$ & $\begin{array}{c}\text { Standard } \\
\text { For SE Combo }\end{array}$ & $\begin{array}{c}\text { 2-Contrast SE } \\
\text { Combo }\end{array}$ & $\begin{array}{c}\text { 3-Contrast SE } \\
\text { Combo }\end{array}$ & $\begin{array}{c}\text { Standard } \\
\text { For FSE Combo }\end{array}$ & $\begin{array}{c}\text { 3-Contrast FSE } \\
\text { Combo }\end{array}$ \\
\hline \hline $\mathrm{T}_{1}$ & 44.679 & 44.699 & 44.684 & 47.878 & 47.006 \\
\hline $\mathrm{PD}$ & 79.828 & - & 79.977 & 79.619 & 79.843 \\
\hline $\mathrm{T}_{2}$ & 15.666 & 15.800 & 15.638 & 17.747 & 17.818 \\
\hline
\end{tabular}

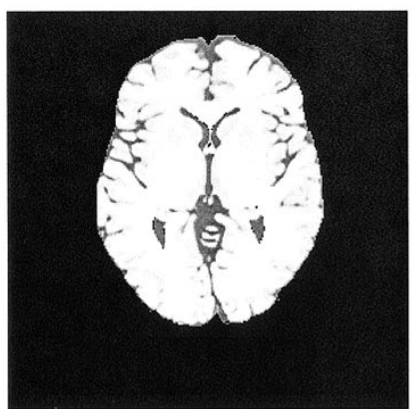

(a)

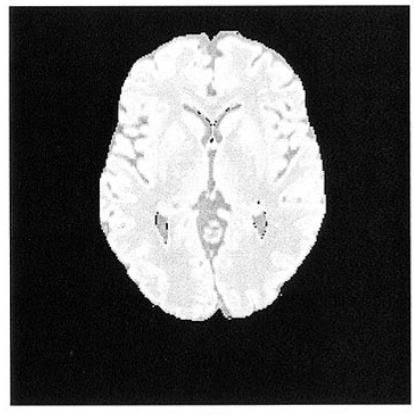

(c)

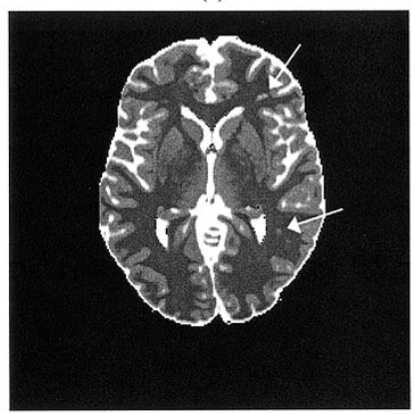

(e)

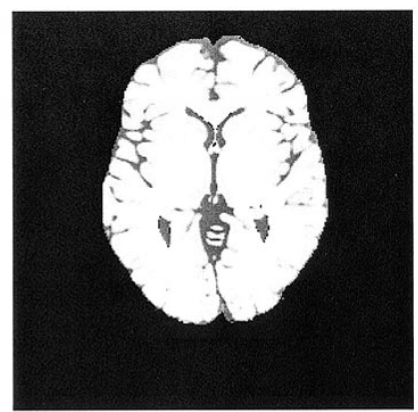

(b)

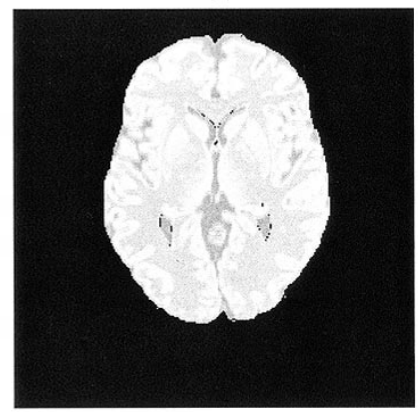

(d)

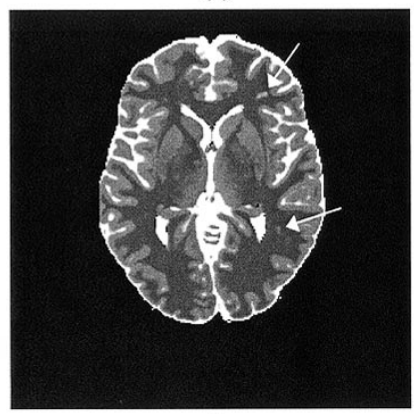

(f)
Fig. 16. Simulated images of phantom data with MS lesions for three different contrasts, $T_{1}$-, PD-, and $T_{2}$-weighted: Images in (a), (c), and (e) from a 3-contrast FSE combo acquisition using the variation scheme in Fig. 10, and images in (b), (d), and (f) from corresponding standard acquisitions with parameters from Table II. Arrows indicate sample locations of MS lesions in (e) and (f), respectively. No difference in visual appearance of MS lesions for images from combo and standard acquisitions is discernible. eral clinical applications, peristaltic and involuntary patient motion are the two most frequently encountered types of motion. The work in this paper aims to achieve scan time reduction with combo acquisitions. Thus, it could be argued that the chance of possible motion during a scan is reduced. This statement holds when a combo acquisition is compared with a complete set of standard acquisitions needed to obtain the same images of different contrasts. The situation is different when the scan time of a combo acquisition is compared with the scan time of each separate standard scan. Typically, the latter is shorter than the former. In general, a comparison is difficult, since there is no separate scan in combo acquisitions. However, in combo acquisitions, segments of low-frequency PE views are separately acquired for each contrast. Therefore, motion occurring during the acquisition of these segments will have the same effect as in standard acquisitions. More specifically, resulting motion artifacts will be limited to one image of a particular contrast. On the other hand, motion artifacts that originated during the acquisition of high-frequency PE views that are shared between multiple images of different contrasts will be visible in all these images. This is obviously not the case in standard acquisitions, where motion artifacts are always confined to the image of a specific contrast being acquired. Hence, the extent of possible "spreads" of motion artifacts into multiple images in combo acquisitions and possible strategies to deal with this problem need further analysis. For example, to reduce the risk of motion artifacts for an image of a particular contrast, combo acquisitions can be designed to acquire all PE views for this contrast at the beginning of the scan, where the occurrence of motion is less likely. In any case, specific aspects of motion artifacts in combo acquisitions only have to be considered for segments of shared $k$-space data, and a possible spread of artifacts into multiple images will always be balanced by the positive effect of overall scan time reduction.

\section{Other Practical Considerations}

The SNR of images from combo and corresponding standard acquisitions with NEX $=1$ was essentially the same (see 
Table XII). In practice, however, different contrast weightings might require different NEX to yield a sufficiently large SNR for reliable diagnosis. Hence, to optimize the SNR of the resulting images from a combo acquisition, a variation scheme for NEX is needed that takes such conditions into account.

Chemical shift artifacts, e.g., water/fat shifts, do not cause problems for the implementation of combo scans introduced in this paper. They are constant for all $\mathrm{PE}$ views in combo acquisitions with variable TE, since in spin echo based imaging these artifacts do not depend on TE, if the bandwidth is kept constant. Similarly, the effect of diffusion will be minor, since no exceptionally large gradient fields are used. In contrast, the inflow of unsaturated spins into selected slices will differ for different PE views due to acquisition with variable TR. Thus, image objects with different spatial distribution of moving spins along the PE direction might show various degrees of inflow enhancement as artifacts of the acquisition method. For small (continuous) changes in TR, these inflow fluctuations will mostly be minor. Finally, the effect of brightening of fat in FSE sequences [44] was not simulated, but would have to be investigated for combo acquisitions in practical applications.

\section{Remaining Challenges}

Despite being assessed by quantitative criteria, the optimization of combo acquisitions was semi-empirical, tissue-specific, and iterative. Moreover, the level of artifacts in the resulting images also depended on the spatial frequency content of the objects being imaged. Although certain robustness of an optimized combo acquisition with respect to multislice imaging and a broad range of relaxation parameters was shown, it remains to be seen how easily combo scans can be applied to different patient populations or anatomy, such as knees or shoulders. A desirable solution would be an automated optimization algorithm that yields optimized parameter variation and PE schemes for any given "patient specific" imaging task. As a prerequisite, a set of quantitative criteria that reliably defines an optimized acquisition for a specified scan time reduction needs to be determined. The challenge is then to derive an algorithm based on these criteria that would select suitable variation and $\mathrm{PE}$ schemes out of the vast space of possible parameter variations of MRI data acquisition.

\section{CONCLUSION}

In this paper, a new technique to reduce clinical MRI scan time and to improve data utilization in multicontrast imaging has been introduced. This technique systematically integrated the acquisition of images of different contrasts into a single scan, using variable acquisition parameters and $k$-space data sharing. Simulations of MRI experiments based on Bloch equations were performed to test the technique of such combo acquisitions using spin echo and fast spin echo sequences. Predicted scan time reductions of $25 \%-50 \%$ compared with standard acquisitions were obtained in simulation studies. Image contrast was well preserved. Artifacts caused by nonuniform $k$-space data weighting were significantly suppressed through semi-empirical optimization of the method. Furthermore, lesion conspicuity was not significantly altered with combo acquisitions. How- ever, artifacts were observed to be dependent on the spatial frequency content and relaxation properties of the objects to be imaged. Signal oscillations due to nonsteady state effects were found to be numerically small, when simulated. In a clinical setting, such combined acquisition also simplifies the protocol setup and increases the scan throughput. In future work, the concept of a combined acquisition of multiple images with different contrast mechanisms will be extended to a more general computerized optimization of protocols including parameter selection schemes, $k$-space sampling, and data sharing in MR imaging.

\section{ACKNOWLEDGMENT}

The authors would like to thank Dr. S. Small for his encouragement and support and the anonymous reviewers for their helpful comments.

\section{REFERENCES}

[1] P. Mansfield, A. A. Maudsley, and T. Baines, "Fast scan proton density imaging by NMR,” J. Phys. E: Scientific. Instrum., vol. 9, p. 271, 1976.

[2] P. S. Melki, F. A. Jolesz, and R. V. Mulkern, "Partial RF echo planar imaging with the FAISE method. I. Experimental and theoretical assessment of artifact," Magn. Reson. Med., vol. 26, pp. 328-341, 1992.

[3] J. Hennig, A. Nauerth, and H. Friedburg, "RARE imaging: A fast imaging method for clinical MR," Magn. Reson. Med., vol. 3, pp. 823-833, 1986

[4] P. van der Meulen, J. P. Groen, and J. J. Cuppen, "Very fast MR imaging by field echoes and small angle excitation," Magn. Reson. Imag., vol. 3, pp. 297-299, 1985.

[5] J. R. MacFall, N. J. Pelc, and R. M. Vavrek, "Correction of spatially dependent phase shifts for partial Fourier imaging," Magn. Reson. Imag., vol. 6, pp. 143-55, 1988.

[6] Z.-P. Liang and P. C. Lauterbur, "An efficient method for dynamic magnetic resonance imaging," IEEE Trans. Med. Imag., vol. 13, pp. 677-686, Dec. 1994.

[7] J. J. van Vaals, M. E. Brummer, W. T. Dixon, H. H. Tuithof, H. Engels, R. C. Nelson, B. M. Gerety, J. L. Chezmar, and J. A. den Boer, "“Keyhole" method for accelerating imaging of contrast agent uptake," J. Magn. Reson. Imag., vol. 3, pp. 671-675, 1993.

[8] R. A. Jones, O. Haraldseth, T. B. Muller, P. A. Rinck, and A. N. Oksendal, "K-space substitution: A novel dynamic imaging technique," Magn. Reson. Med., vol. 29, pp. 830-834, 1993.

[9] X. Hu and T. Parrish, "Reduction of field of view for dynamic imaging," Magn. Reson. Med., vol. 31, pp. 691-694, 1994.

[10] G. P. Zientara, L. P. Panych, and F. A. Jolesz, "Dynamically adaptive MRI with encoding by singular value decomposition," Magn. Reson. Med., vol. 32, pp. 268-274, 1994.

[11] B. Madore, G. H. Glover, and N. J. Pelc, "Unaliasing by Fourier-encoding the overlaps using the temporal dimension (UNFOLD), applied to cardiac imaging and fMRI," Magn. Reson. Med., vol. 42, pp. 813-828, 1999.

[12] D. K. Sodickson and W. J. Manning, "Simultaneous acquisition of spatial harmonics (SMASH): Fast imaging with radiofrequency coil arrays," Magn. Reson. Med., vol. 38, pp. 591-603, 1997.

[13] K. P. Pruessmann, M. Weiger, M. B. Scheidegger, and P. Boesiger, "SENSE: Sensitivity encoding for fast MRI," Magn. Reson. Med., vol. 42, pp. 952-962, 1999.

[14] D. B. Twieg, "The $k$-trajectory formulation of the NMR imaging process with applications in analysis and synthesis of imaging methods," Med. Phys., vol. 10, pp. 610-621, 1983.

[15] S. Ljunggren, "A simple graphical representation of Fourier-based imaging methods," J. Magn. Reson., vol. 54, pp. 338-343, 1983.

[16] C. H. Meyer, B. S. Hu, D. G. Nishimura, and A. Macovski, "Fast spiral coronary artery imaging," Magn. Reson. Med., vol. 28, pp. 202-213, 1992.

[17] V. Rasche, D. Holz, and S. W., "Radial turbo spin echo imaging," Magn. Reson. Med., vol. 32, pp. 629-638, 1994.

[18] S. J. Norton, "Fast magnetic resonance imaging with simultaneously oscillating and rotating field gradients," IEEE Trans. Med. Imag., vol. MI-6, pp. 21-31, 1987. 
[19] L. P. Panych, R. V. Mulkern, P. Saiviroonporn, G. P. Zientara, and F A. Jolesz, "Non-Fourier encoding with multiple spin echoes," Magn. Reson. Med., vol. 38, pp. 964-973, 1997.

[20] R. K. Butts, F. Farzaneh, S. J. Riederer, J. N. Rydberg, and R. C. Grimm, "T2-weighted spin-echo pulse sequence with variable repetition and echo times for reduction of MR image acquisition time," Radiology, vol. 180, pp. 551-556, 1991.

[21] H. K. Song and F. W. Wehrli, "Variable TE gradient and spin echo sequences for in vivo MR microscopy of short T2 species," Magn. Reson. Med., vol. 39, pp. 251-258, 1998 .

[22] W. Lin, E. M. Haacke, and J. A. Tkach, "Three-dimensional time-offlight MR angiography with variable TE (VARIETE) for fat signal reduction," Magn. Reson. Med., vol. 32, pp. 678-683, 1994.

[23] R. T. Constable and J. C. Gore, "The loss of small objects in variable TE imaging: Implications for FSE, RARE, and EPI," Magn. Reson. Med., vol. 28, pp. 9-24, 1992.

[24] J. A. Tkach, W. Lin, J. J. J. Duda, E. M. Haacke, and T. J. Masaryk, “Optimizing three-dimensional time-of-flight MR angiography with variable repetition time," Radiology, vol. 191, pp. 805-811, 1994.

[25] B. Kuhn, W. Dreher, D. G. Norris, and D. Leibfritz, "Fast proton spectroscopic imaging employing $k$-space weighting achieved by variable repetition times," Magn. Reson. Med., vol. 35, pp. 457-464, 1996.

[26] X. Hu, M. Patel, W. Chen, and K. Ugurbil, "Reduction of truncation artifacts in chemical-shift imaging by extended sampling using variable repetition time," J. Magn. Reson. Ser. B, vol. 106, pp. 292-296, 1995.

[27] D. A. Feinberg, B. Kiefer, and A. W. Litt, "Dual contrast GRASE (gradient-spin echo) imaging using mixed bandwidth," Magn. Reson. Med., vol. 31, pp. 461-464, 1994.

[28] F. G. Woermann, H. Steiner, G. J. Barker, P. A. Bartlett, C. E. Elger, J. S. Duncan, and M. R. Symms, "A fast FLAIR dual-echo technique for hippocampal T2 relaxometry: First experiences in patients with temporal lobe epilepsy," J. Magn. Reson. Imag., vol. 13, pp. 547-552, 2001.

[29] N. Higuchi, K. Oshio, M. S., H. Shiga, P. S. Melki, R. V. Mulkern, and F. A. Jolesz, "Two contrast RARE: A fast spin-density and T2-weighted imaging method," J. Magn. Reson. Imag., vol. 1, p. 147, 1991.

[30] H. Bruder, H. Fischer, R. Graumann, and M. Deimling, "A new steadystate imaging sequence for simultaneous acquisition of two MR images with clearly different contrasts," Magn. Reson. Med., vol. 7, pp. 35-42, 1988.

[31] K. Oshio and F. A. Jolesz, "Simultaneous acquisition of proton density, T1, and T2 images with triple contrast RARE sequence," J. Comput. Assist. Tomogr., vol. 17, pp. 333-338, 1993.
[32] B. A. Johnson, E. K. Fram, B. P. Drayer, B. L. Dean, P. J. Keller, and R. Jacobowitz, "Evaluation of shared-view acquisition using repeated echoes (SHARE): A dual-echo fast spin-echo MR technique," AJNR: Amer. J. Neuroradiol., vol. 15, pp. 667-673, 1994.

[33] S. J. Riederer, T. Tasciyan, F. Farzaneh, J. N. Lee, R. C. Wright, and R. J. Herfkens, "MR fluoroscopy: Technical feasibility," Magn. Reson. Med., vol. 8, pp. 1-15, 1988.

[34] F. Bloch, "Nuclear induction," Physical Rev., vol. 70, pp. 460-74, 1946.

[35] R. K. Kwan, A. C. Evans, and G. B. Pike, "MRI simulation-based evaluation of image-processing and classification methods," IEEE Trans. Med. Imag., vol. 18, pp. 1085-1097, Nov. 1999.

[36] D. L. Collins, A. P. Zijdenbos, V. Kollokian, J. G. Sled, N. J. Kabani, C. J. Holmes, and A. C. Evans, "Design and construction of a realistic digital brain phantom," IEEE Trans. Med. Imag., vol. 17, pp. 463-468, June 1998.

[37] E. M. Haacke, R. W. Brown, M. R. Thompson, and R. Venkatesan, Magnetic Resonance Imaging: Physical Principles and Sequence Design. New York: Wiley, 1999.

[38] P. S. Melki, R. V. Mulkern, L. P. Panych, and F. A. Jolesz, "Comparing the FAISE method with conventional dual-echo sequences," J. Magn. Reson. Imag., vol. 1, pp. 319-326, 1991.

[39] J. Listerud, S. Einstein, E. Outwater, and H. Y. Kressel, "First principles of fast spin echo," Magn. Reson. Quart., vol. 8, pp. 199-244, 1992.

[40] A. Macovski, "Noise in MRI," Magn. Reson. Med., vol. 36, pp. 494-497, 1996.

[41] E. R. McVeigh, R. M. Henkelman, and M. J. Bronskill, "Noise and filtration in magnetic resonance imaging," Med. Phys., vol. 12, pp. 586-591, 1985.

[42] H. Gudbjartsson and S. Patz, "The Rician distribution of noisy MRI data," Magn. Reson. Med., vol. 34, pp. 910-914, 1995.

[43] S. Barbosa, L. D. Blumhardt, N. Roberts, T. Lock, and R. H. Edwards, "Magnetic resonance relaxation time mapping in multiple sclerosis: Normal appearing white matter and the "invisible" lesion load," Magn Reson. Imag., vol. 12, pp. 33-42, 1994.

[44] R. M. Henkelman, P. A. Hardy, J. E. Bishop, C. S. Poon, and D. B. Plewes, "Why fat is bright in RARE and fast spin-echo imaging," $J$. Magn. Reson. Imag., vol. 2, pp. 533-540, 1992. 